\title{
AEGIS-X: DEEP CHANDRA IMAGING OF THE CENTRAL GROTH STRIP
}

\author{
K. Nandra ${ }^{1,2}$, E. S. Laird ${ }^{2}$, J. A. Aird ${ }^{3,4}$, M. Salvato $^{1}$, A. Georgakakis ${ }^{1}$, G. Barro ${ }^{5,6}$, P. G. Perez-Gonzalez ${ }^{6}$, P. Barmby ${ }^{7}$,

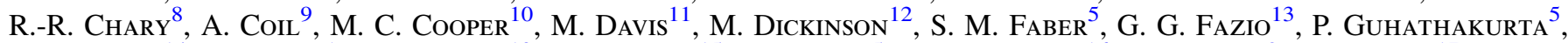 \\ S. GWYN ${ }^{14}$, L.-T. Hsu ${ }^{1}$, J.-S. HuANG ${ }^{10}$, R. J. Ivison ${ }^{15}$, D. C. Koo ${ }^{5}$, J. A. NewMAN ${ }^{16}$, C. RANGEL ${ }^{2}$, T. YAMADA ${ }^{17}$, AND \\ C. Willmer ${ }^{18}$ \\ ${ }^{1}$ Max Planck Institute for Extraterrestrial Physics, Giessenbachstrasse, D-85741 Garching, Germany \\ ${ }^{2}$ Astrophysics Group, Blackett Laboratory, Imperial College London, London SW7 2AZ, UK \\ ${ }^{3}$ Center for Astrophysics and Space Science, University of California, San Diego, CA 92093, USA \\ ${ }^{4}$ Department of Physics, University of Durham, Durham DH1, 3LE, UK \\ ${ }^{5}$ UCO/Lick Observatory, Department of Astronomy and Astrophysics, University of California, Santa Cruz, CA 95064, USA \\ ${ }^{6}$ Departamento de Astrofísica, Facultad de CC. Físicas, Universidad Complutense de Madrid, E-28040 Madrid, Spain \\ ${ }^{7}$ Department of Physics and Astronomy, University of Western Ontario, London, Ontario N6A 3K7, Canada \\ ${ }^{8}$ MS220-6, Caltech, Pasadena, CA 91125, USA \\ 9 Department of Physics, Center for Astrophysics and Space Sciences, University of California at San Diego, 9500 Gilman Dr., La Jolla, San Diego, CA 92093, USA \\ ${ }^{10}$ Center for Galaxy Evolution, Department of Physics and Astronomy, University of California, Irvine, CA 92697, USA \\ ${ }^{11}$ Department of Astronomy, University of California, Berkeley, CA 94720, USA \\ ${ }^{12}$ NOAO, Tucson, AZ 85719, USA \\ ${ }^{13}$ Harvard-Smithsonian Center for Astrophysics, Cambridge, MA 02138, USA \\ ${ }^{14}$ CADC, HIA, Victoria, Canada \\ ${ }^{15}$ Institute for Astronomy, University of Edinburgh, Royal Observatory, Blackford Hill, Edinburgh EH9 3HJ, UK \\ ${ }^{16}$ University of Pittsburgh and Pitt-PACC, 3941 O'Hara St., Pittsburgh, PA 15260, USA \\ ${ }^{17}$ Tohoku University, Aramaki, Aoba, Sendai 9808578, Japan \\ ${ }^{18}$ Steward Observatory, University of Arizona, 933 North Cherry Avenue, Tucson, AZ 85721, USA \\ Received 2014 April 8; accepted 2015 March 29; published 2015 September 9
}

\begin{abstract}
We present the results of deep Chandra imaging of the central region of the Extended Groth Strip, the AEGIS-X Deep (AEGIS-XD) survey. When combined with previous Chandra observations of a wider area of the strip, AEGIS-X Wide (AEGIS-XW), these provide data to a nominal exposure depth of $800 \mathrm{ks}$ in the three central ACISI fields, a region of approximately $0.29 \mathrm{deg}^{2}$. This is currently the third deepest X-ray survey in existence; a factor $\sim 2-3$ shallower than the Chandra Deep Fields (CDFs), but over an area $\sim 3$ times greater than each CDF. We present a catalog of 937 point sources detected in the deep Chandra observations, along with identifications of our X-ray sources from deep ground-based, Spitzer, GALEX, and Hubble Space Telescope imaging. Using a likelihood ratio analysis, we associate multiband counterparts for 929/937 of our X-ray sources, with an estimated 95\% reliability, making the identification completeness approximately $94 \%$ in a statistical sense. Reliable spectroscopic redshifts for 353 of our X-ray sources are available predominantly from Keck (DEEP2/3) and MMT Hectospec, so the current spectroscopic completeness is $\sim 38 \%$. For the remainder of the X-ray sources, we compute photometric redshifts based on multiband photometry in up to 35 bands from the UV to mid-IR. Particular attention is given to the fact that the vast majority the $\mathrm{X}$-ray sources are active galactic nuclei and require hybrid templates. Our photometric redshifts have mean accuracy of $\sigma=0.04$ and an outlier fraction of approximately 5\%, reaching $\sigma=0.03$ with less than $4 \%$ outliers in the area covered by CANDELS . The X-ray, multiwavelength photometry, and redshift catalogs are made publicly available.
\end{abstract}

Key words: galaxies: active - galaxies: nuclei - surveys

Supporting material: machine-readable tables

\section{INTRODUCTION}

Deep X-ray surveys trace the accretion history of the universe, offering a highly efficient method of pinpointing growing black holes in galaxies across a wide range of redshifts. Chandra and XMM-Newton surveys have yielded samples of active galactic nuclei (AGNs) capable of characterizing the evolution of accretion power in the universe, via the X-ray luminosity function (XLF; e.g., Barger et al. 2005; Hasinger et al. 2005; Silverman et al. 2008; Aird et al. 2010; Ueda et al. 2014). These surveys have also been highly influential in broadening our understanding of the co-evolution of supermassive black holes and galaxies via the characterization of various host properties of AGN. The extraordinary sensitivity of the current generation of X-ray observatories, particularly Chandra, to point-like X-ray sources has transformed such investigations by revealing large populations of AGN in galaxies where the accretion activity in other wavebands is either obscured or overwhelmed by host galaxy light (e.g., Brandt \& Hasinger 2005).

Thus far, the deepest X-ray surveys are the Chandra Deep Fields (CDFs; Giacconi et al. 2002; Alexander et al. 2003; Luo et al. 2010; Xue et al. 2011). While these reach extremely faint flux levels, they can only do so over relatively small areas. Complementary large area, but shallower surveys have therefore been performed, such as XBootes (Murray et al. 2005), XMM-LSS (Pierre et al. 2007), XMM-COSMOS (Hasinger et al. 2007), and Chandra COSMOS (Elvis et al. 2009).

Determination of the accretion history and its relationship to galaxy evolution cannot be achieved using X-ray data alone. For example, it is also a basic requirement to determine the redshifts of the X-ray sources to calculate their luminosities and 
Table 1

Observation Log

\begin{tabular}{|c|c|c|c|c|c|c|c|}
\hline $\begin{array}{l}\text { Field }^{\mathrm{a}} \\
\text { Name }\end{array}$ & ObsID $^{b}$ & $\begin{array}{l}\text { R.A. }^{\text {c }} \\
(\text { J2000) }\end{array}$ & $\begin{array}{c}\text { Decl. }^{\mathrm{c}} \\
(\mathrm{J} 2000)\end{array}$ & $\begin{array}{c}\text { Start Time } \\
\text { (UT) }\end{array}$ & $\begin{array}{c}\text { On Time } \\
(\mathrm{ks})\end{array}$ & $\begin{array}{c}\text { Exposure }^{\mathrm{f}} \\
(\mathrm{ks})\end{array}$ & $\begin{array}{c}\text { Roll Angle } \\
\text { (deg) }\end{array}$ \\
\hline AEGIS-1 & 9450 & 142017.24 & +5300 34.22 & 2007 Dec 11 04:24:07 & 28.91 & 28.78 & 40.2 \\
\hline AEGIS-1 & 9451 & 142017.23 & +530034.23 & 2007 Dec 16 10:52:06 & 25.38 & 25.21 & 40.2 \\
\hline AEGIS-1 & 9452 & 142016.80 & +530036.20 & 2007 Dec 18 05:45:49 & 13.56 & 13.29 & 48.7 \\
\hline AEGIS-1 & 9453 & 142014.22 & +525943.05 & 2008 Jun 15 21:28:03 & 44.75 & 44.64 & 229.2 \\
\hline AEGIS-1 & 9720 & 142014.21 & +525942.96 & 2008 Jun 17 05:14:02 & 28.14 & 27.74 & 229.2 \\
\hline AEGIS-1 & 9721 & 142013.78 & +525945.13 & 2008 Jun 12 08:09:14 & 16.55 & 16.55 & 220.2 \\
\hline AEGIS-1 & 9722 & 142013.76 & +525945.19 & 2008 Jun 13 07:02:28 & 20.01 & 19.89 & 220.2 \\
\hline AEGIS-1 & 9723 & 142014.22 & +525943.00 & 2008 Jun 18 13:42:40 & 34.54 & 34.47 & 229.2 \\
\hline AEGIS-1 & 9724 & 142016.82 & +530036.22 & 2007 Dec 22 13:37:26 & 14.09 & 14.08 & 48.7 \\
\hline AEGIS-1 & 9725 & 142012.35 & +530016.04 & 2008 Mar 31 05:21:42 & 31.13 & 31.13 & 150.2 \\
\hline AEGIS-1 & 9726 & 142013.77 & +525945.15 & 2008 Jun 05 08:45:04 & 39.63 & 39.62 & 220.2 \\
\hline AEGIS-1 & 9793 & 142016.81 & +530036.27 & 2007 Dec 19 02:53:51 & 24.08 & 23.83 & 48.7 \\
\hline AEGIS-1 & 9794 & 142016.82 & +530036.13 & 2007 Dec 20 04:27:59 & 10.34 & 10.03 & 48.7 \\
\hline AEGIS-1 & 9795 & 142016.82 & +530036.32 & 2007 Dec 20 21:36:20 & 8.91 & 8.91 & 48.7 \\
\hline AEGIS-1 & 9796 & 142016.81 & +530036.27 & 2007 Dec 21 20:28:33 & 16.33 & 16.33 & 48.7 \\
\hline AEGIS-1 & 9797 & 142016.82 & +530036.38 & 2007 Dec 23 13:12:28 & 12.63 & 12.15 & 48.7 \\
\hline AEGIS-1 & 9842 & 142012.35 & +530016.01 & 2008 Apr 02 21:01:59 & 30.45 & 30.44 & 150.2 \\
\hline AEGIS-1 & 9843 & 142012.34 & +530016.13 & 2008 Apr 02 01:11:09 & 15.32 & 13.48 & 150.2 \\
\hline AEGIS-1 & 9844 & 142012.35 & +530015.94 & 2008 Apr 05 13:07:54 & 19.78 & 19.78 & 150.2 \\
\hline AEGIS-1 & 9863 & 142013.76 & +525945.14 & 2008 Jun 07 00:33:47 & 22.01 & 22.01 & 220.2 \\
\hline AEGIS-1 & 9866 & 142013.77 & +525945.16 & 2008 Jun 03 22:43:14 & 25.83 & 25.83 & 220.2 \\
\hline AEGIS-1 & 9870 & 142013.77 & +525944.99 & 2008 Jun 10 15:11:23 & 11.08 & 11.00 & 220.2 \\
\hline AEGIS-1 & 9873 & 142013.77 & +525945.16 & 2008 Jun 11 14:22:06 & 30.81 & 30.75 & 220.2 \\
\hline AEGIS-1 & 9875 & 142014.32 & +525942.61 & 2008 Jun 23 22:54:14 & 25.21 & 25.20 & 231.2 \\
\hline AEGIS-1 & 9876 & 142014.21 & +525943.03 & 2008 Jun 22 00:22:03 & 33.29 & 33.28 & 229.2 \\
\hline AEGIS-2 & 9454 & 141914.72 & +524822.75 & 2008 Sep 11 04:47:10 & 59.81 & 56.80 & 310.7 \\
\hline AEGIS-2 & 9455 & 141914.72 & +524822.78 & 2008 Sep 13 19:38:46 & 100.20 & 99.72 & 310.7 \\
\hline AEGIS-2 & 9456 & 141915.06 & +524829.45 & 2008 Sep 24 08:15:30 & 58.82 & 58.35 & 325.2 \\
\hline AEGIS-2 & 9457 & 141911.06 & +524810.35 & 2008 Jun 27 07:08:38 & 32.75 & 32.74 & 235.7 \\
\hline AEGIS-2 & 9727 & 141914.72 & +524822.82 & 2008 Sep 12 16:44:12 & 36.16 & 34.94 & 310.7 \\
\hline AEGIS-2 & 9729 & 141911.31 & +524809.80 & 2008 Jul 09 16:47:58 & 48.29 & 48.04 & 240.2 \\
\hline AEGIS-2 & 9730 & 141915.06 & +524829.41 & 2008 Sep 25 16:50:54 & 53.97 & 53.72 & 325.2 \\
\hline AEGIS-2 & 9731 & 141911.25 & +524809.91 & 2008 Jul 03 10:58:47 & 21.38 & 21.38 & 239.2 \\
\hline AEGIS-2 & 9733 & 141915.06 & +524829.44 & 2008 Sep 27 01:15:33 & 58.82 & 58.36 & 325.2 \\
\hline AEGIS-2 & 9878 & 141911.07 & +524810.42 & 2008 Jun 28 06:03:20 & 15.74 & 15.73 & 235.7 \\
\hline AEGIS-2 & 9879 & 141911.07 & +524810.36 & 2008 Jun 29 03:39:20 & 27.02 & 26.80 & 235.7 \\
\hline AEGIS-2 & 9880 & 141911.25 & +524809.87 & 2008 Jul 05 17:00:17 & 29.89 & 29.45 & 239.2 \\
\hline AEGIS-2 & 9881 & 141915.06 & +524829.47 & 2008 Sep 28 08:15:12 & 54.53 & 53.93 & 325.2 \\
\hline AEGIS-3 & 9458 & 141806.09 & +523717.03 & 2009 Mar 18 12:20:16 & 6.66 & 6.65 & 136.9 \\
\hline AEGIS-3 & 9459 & 141812.11 & +523657.84 & 2008 Sep 30 19:20:28 & 69.91 & 69.55 & 329.7 \\
\hline AEGIS-3 & 9460 & 141812.12 & +523658.08 & 2008 Oct 10 06:17:49 & 21.91 & 21.36 & 330.2 \\
\hline AEGIS-3 & 9461 & 141807.78 & +523637.50 & 2009 Jun 26 09:30:12 & 23.73 & 23.73 & 230.2 \\
\hline AEGIS-3 & 9734 & 141811.83 & +523650.93 & 2008 Sep 16 11:01:21 & 49.98 & 49.47 & 315.2 \\
\hline AEGIS-3 & 9735 & 141811.83 & +523650.95 & 2008 Sep 19 03:14:15 & 50.00 & 49.47 & 315.2 \\
\hline AEGIS-3 & 9736 & 141811.83 & +523650.97 & 2008 Sep 20 11:07:10 & 50.13 & 49.48 & 315.2 \\
\hline AEGIS-3 & 9737 & 141811.83 & +523650.94 & 2008 Sep 21 17:53:00 & 49.99 & 49.48 & 315.2 \\
\hline AEGIS-3 & 9738 & 141812.11 & +523657.84 & 2008 Oct 02 06:56:22 & 61.89 & 60.60 & 329.7 \\
\hline AEGIS-3 & 9739 & 141812.12 & +523658.09 & 2008 Oct 05 11:28:12 & 42.91 & 42.59 & 330.2 \\
\hline AEGIS-3 & 9740 & 141806.30 & +523719.96 & 2009 Mar 09 22:24:18 & 20.38 & 20.37 & 130.2 \\
\hline AEGIS-3 & 10769 & 141805.99 & +523714.25 & 2009 Mar 20 13:38:26 & 26.69 & 26.68 & 143.0 \\
\hline AEGIS-3 & 10847 & 141809.85 & +523732.39 & 2008 Dec 31 05:06:27 & 19.27 & 19.27 & 57.2 \\
\hline AEGIS-3 & 10848 & 141809.86 & +523732.43 & 2009 Jan 01 17:11:57 & 17.91 & 17.91 & 57.2 \\
\hline AEGIS-3 & 10849 & 141809.85 & +523732.52 & 2009 Jan 02 21:25:57 & 15.93 & 15.92 & 57.2 \\
\hline AEGIS-3 & 10876 & 141806.30 & +523719.99 & 2009 Mar 11 01:37:20 & 17.21 & 17.21 & 130.2 \\
\hline AEGIS-3 & 10877 & 141806.31 & +523720.03 & 2009 Mar 12 15:15:57 & 16.23 & 16.22 & 130.2 \\
\hline AEGIS-3 & 10896 & 141807.50 & +523638.72 & 2009 Jun 15 18:46:14 & 23.29 & 23.29 & 224.7 \\
\hline AEGIS-3 & 10923 & 141807.77 & +523637.40 & 2009 Jun 22 07:38:22 & 11.62 & 11.62 & 230.2 \\
\hline
\end{tabular}

Notes.

${ }^{a}$ Field name: note that there is an approximate correspondence between, respectively, AEGIS1-3 and EGS 3-5 in the AEGIS-XW survey of L09.

b Chandra observation ID.

c Nominal position of pointing (J2000).

${ }^{\mathrm{d}}$ Start date and time (UT). 
e Raw exposure time.

${ }^{\mathrm{f}}$ Exposure time after data screening as described in Section 2.2.

$\mathrm{g}$ Roll angle in deg of the observation.

evolution. This has proved surprisingly difficult for a number of reasons. First, the depth of current X-ray observations is such that the multiwavelength counterparts of the X-ray sources are often extremely faint. This makes even the identification of optical or near-infrared (NIR) counterparts challenging. Determining their redshifts is even more difficult because the vast majority are too faint for spectroscopic identification. Despite major efforts, the spectroscopic completeness of the deepest X-ray samples are $<50 \%$ (e.g., Szokoly et al. 2004; Trouille et al. 2008). Photometric redshifts can be used to mitigate this spectroscopic incompleteness, but require very deep data in as many bands as possible. This can be difficult to acquire in wide fields. Also, because the vast majority of X-ray point sources in deep surveys are AGN, special consideration is required to yield accurate photo- $z$ (Salvato et al. 2009, 2011).

One area of the sky that benefits from deep multiwavelength coverage is the Extended Groth Strip (EGS), which is a region of $0.25 \times 2^{\prime}$ centered at approximately $\alpha=14^{\mathrm{h}} 18^{\mathrm{m}}$, $\delta=52^{\circ} 00^{\prime}$. Deep observations of the EGS have been performed using ground- and space-based observatories, many as part of the AEGIS multiwavelength project (Davis et al. 2007). This includes X-ray imaging with Chandra/ACIS, which covers the entire EGS to a nominal depth of $200 \mathrm{ks}$; we henceforth designate this the AEGIS-X Wide (AEGIS-XW) survey (Nandra et al. 2005; Laird et al. 2009 hereafter L09). These observations have been effective in helping to characterize the accretion history to relatively high redshifts (Aird et al. 2008, 2010). In combination with the AEGIS multiwavelength data, they have also provided new insights into the relationship of AGN with their host galaxies (e.g., Nandra et al. 2007; Pierce et al. 2007; Bundy et al. 2008; Georgakakis et al. 2008c, 2009) and large-scale structure environments (e.g., Georgakakis et al. 2007, 2008a; Coil et al. 2009).

In the current paper we present deeper Chandra imaging (800 ks nominal depth) of the central EGS region, hereafter the AEGIS-X Deep (AEGIS-XD) survey. The AEGIS-XW data are sufficient to detect essentially the full population of X-ray emitting AGN to $z \sim 3$ (Aird et al. 2008). Understanding the evolution above this redshift is important, because above $z=3$ the luminous QSO population is known to decline rapidly (e.g., Wall et al. 2005). Whether this also applies to the obscured and more moderate luminosity AGN populations probed in X-ray surveys is an open question (Brusa et al. 2009; Civano et al. 2011; Vito et al. 2013). The CDFs reach sufficient depths to detect such sources at $z>3$ (e.g., Fiore et al. 2012), but do not cover enough area to yield samples of sufficient size to fully characterize the total accretion power at the redshifts of interest. A further advantage of deeper X-ray data is the improved characterization of the X-ray spectral properties of deep field sources, and particularly the obscuration properties, which may be strongly linked to SMBH-galaxy co-evolution scenarios (e.g., Hopkins et al. 2005). Because most Chandra surveys are severely photon-starved, this is very challenging without long exposures.

The AEGIS-XD data occupy a unique region of parameter space because they are larger in area than each CDF by a factor 3 , but of sufficient depth to probe the high redshift and obscured X-ray source populations of interest. The AEGIS-XW data were designed to probe down to Seyfert-level AGN activity $\left(L_{\mathrm{X}} \sim 10^{43} \mathrm{erg} \mathrm{s}^{-1}\right.$ at $z \sim 3$ in the soft $\mathrm{X}$-ray $(0.5-2 \mathrm{keV})$ band). The new AEGIS-XD observations are sensitive to these luminosities at $z \sim 5$ in principle, with sufficient area to extend our understanding of the evolution of the XLF to at least that redshift. At the same time, in the harder $X$-ray band $(2-10 \mathrm{keV})$, the AEGIS-XD images are sensitive to these kinds of Seyfert-level luminosities at $z \sim 2$, even for sources absorbed by a column density of $N_{\mathrm{H}}=10^{24} \mathrm{~cm}^{-2}$. At these flux limits, sources with even higher column densities may be detected via their scattered light in either or both bands via consideration of their X-ray spectra (e.g., Tozzi et al. 2006; Brightman \& Ueda 2012). When combined with the exceptional multiwavelength data in AEGIS, the AEGIS-XD data provide a unique resource to trace the growth of supermassive black holes, and their influence on galaxies, over a major fraction of cosmic time.

In the present paper we describe the basic observational parameters and analysis of the AEGIS-XD data, deriving an $\mathrm{X}$-ray point source catalog, a multiwavelength photometric catalog of the X-ray source counterparts, and a redshift catalog. A companion paper presenting an extended source catalog based on the same deep Chandra data was presented in Erfanianfar et al. (2013). An X-ray point source catalog based on almost the same Chandra data used here was previously presented by Goulding et al. (2012; hereafter G12). The present paper employs a different data reduction and source detection procedure, as well as a different technique for association of optical and NIR counterparts to the X-ray sources. Comparisons with the previous work are given in Sections 3.3 and 4.2. In addition, we provide a catalog of spectroscopic and photometric redshifts for the X-ray source counterparts. The paper is structured as follows: Section 2 describes the X-ray observations and data reduction. The point source catalog and sensitivity maps are presented in Section 3. Section 4 describes the multiwavelength identifications and multiband photometry, the latter based on the methodology of the Rainbow database (Pérez-González et al. 2008; Barro et al. 2011a, 2011b). In Section 5 we present redshift estimates of the sources, including accurate photometric redshifts accounting for the AGN nature of the sources using the methods of Salvato et al. (2011). A summary of our results is given in Section 6.

\section{OBSERVATIONS AND DATA REDUCTION}

\subsection{X-ray Data}

The new AEGIS-XD Chandra data were taken at three nominal pointing positions, which we have designated AEGIS1, AEGIS-2, and AEGIS-3. Each field was approved to receive approximately $600 \mathrm{ks}$ in exposure as part of Chandra Cycle 9, supplementing the $\sim 200 \mathrm{ks}$ exposures acquired in Cycle 3 (Nandra et al. 2005) and Cycle 6 (L09). The new exposures were split up into smaller segments ranging in duration from $\sim 7$ to $100 \mathrm{ks}$. These observations were all taken in the time period 2007 December 11 to 2009 June 26 using the ACIS-I instrument (Garmire et al. 2003) without any grating in place. All new AEGIS-XD data were taken in VFAINT mode. Full 
Table 2

AEGIS-XW 200 ks ObsIDs Combined with New AEGIS-XD Fields (Table 1)

\begin{tabular}{lc}
\hline \hline AEGIS-XD Field & AEGIS-XW 200 ks ObsIDs \\
\hline AEGIS-1 & $5845,5846,6214,6215$ \\
AEGIS-2 & $5847,5848,6216,6217$ \\
AEGIS-3 & $3305,4357,4365,5849,5850,6218,6219$
\end{tabular}

details of the new Chandra observations are given in Table 1. The three subfields AEGIS-1, 2, and 3 were defined as the spatial limits of ObsIDs 9450, 9454, and 9458, respectively, with a border of \pm 20 pixels in the $X$ and $Y$ directions. These subfields were analyzed separately but have considerable overlap; common sources were removed at a later stage.

The total exposure times for the Cycle 9 pointings before screening were $583 \mathrm{ks}$ (AEGIS-1), $597 \mathrm{ks}$ (AEGIS-2), and $596 \mathrm{ks}$ (AEGIS-3). The centers of these fields correspond fairly closely to those of the EGS-3, EGS-4, and EGS-5 fields of L09. Here we combine the data from the 200 ks EGS 3-5 fields with our new data, to a nominal $800 \mathrm{ks}$ depth in each field. For the AEGIS-1 sub-field there is some overlap with the EGS2 data of L09, but this is very small and at large off-axis angles, so these data were not included. For the AEGIS-3 sub-field, we also add three ObsIDs from the original "Groth-Westphal Strip" (GWS) survey of Nandra et al. (2005), which were taken in FAINT mode. These have significantly different pointing centers and roll angles to the L09 AEGIS-XW survey, but there is substantial overlap of the tiles with the AEGIS-3 field at one edge. A list of the ObsIDs that overlapped with each field is given in Table 2. Note that the final catalog presented here consists only of the sources in the region that is covered by the new X-ray imaging (i.e., covered by the ObsIDs listed in Table 1), otherwise they should be present in the catalog of L09.

The total (pre-screening) exposure times for each field were $779 \mathrm{ks}$ (AEGIS-1), $787 \mathrm{ks}$ (AEGIS-2), and $782 \mathrm{ks}$ (AEGIS-3, excluding the GWS/EGS-8 exposure, which only covers a relatively small part of the field). The data and analysis of the $200 \mathrm{ks}$ imaging of the AEGIS-XW survey is fully described in L09. Here we largely follow the same reduction analysis methodology described in that paper, which we now describe in brief, noting any changes.

\subsection{X-Ray Data Reduction}

The data reduction was performed using the CIAO data analysis software v4.1.2 (Fruscione et al. 2009) and follows the scheme described in L09, with some minor changes and improvements. Briefly, for each individual Obsid, we corrected the data for aspect offsets, applied the bad pixel removal and destreaking algorithms, removed cosmic ray afterglows using standard tools, and corrected for CTI and gain effects. We also applied the ACIS particle background cleaning algorithm to the VFAINT mode data. Analysis was restricted to ACIS chips 0-3 and ASCA-style event grades 0, 2, 4, and 6. To reject periods of high background, we used the procedure of Nandra et al. (2007), adopting a threshold of 1.4 times the quiescent background level, determined as the count rate at which the background shows zero excess variance over that expected from statistical fluctuations alone. As in L09 and Nandra et al. (2005) we relaxed this criterion in ObsID 4365, which contains a period of relatively high but stable background. As in L09, a flare was also manually removed from ObsID 5850.

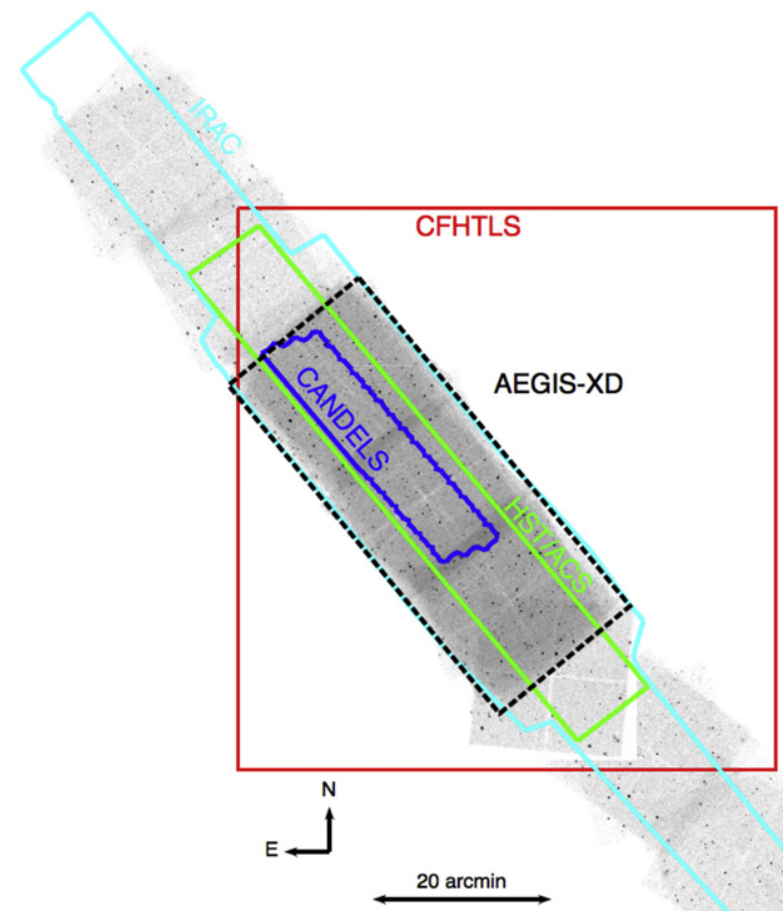

Figure 1. Layout of the AEGIS field showing the location of the Chandra $\mathrm{X}$-ray imaging and a subset of the multiwavelength coverage. The $200 \mathrm{ks}$ AEGIS-XW area (L09) is shown as a grayscale image. The deeper $800 \mathrm{ks}$ coverage is contained in the area delineated within the thick black lines.

Following this basic reduction and screening, the astrometry of the individual image frames was corrected using a reference catalog. Specifically, we use the CFHTLS $i$-band selected catalog to register the AEGIS-XD images to the optical reference frame. We first ran the Chandra wavelet source detection task wavdetect on the $0.5-7 \mathrm{keV}$ image, using a detection threshold of $10^{-6}$, and then used the CIAO task reproject_aspect to correct the astrometry compared to the reference image to create new aspect solution files. The new aspect solutions were then applied to the original event files. The parameters used for reproject_aspect were a source match radius of 120 and a residual limit of 0.50 . Typically, around 100 sources were detected in the individual ObsIDs, with around $60 \%$ of these having a counterpart used in the reprojection. The absolute value of the offset was typically small $(<0$ ". 5). Following this step we created event files for the individual frames in the standard full (FB; $0.5-7 \mathrm{keV})$, soft (SB; $0.5-2 \mathrm{keV}$ ), hard (HB; $2-7 \mathrm{keV})$, and ultrahard (UB; 4-7 keV) bands; exposure maps for each were produced for each energy band using the merge_all task. The exposure maps were created at multiple energies with weights appropriate for a $\Gamma=1.4$ power-law spectrum (see Table 3 ). The individual ObsID images and exposure maps were then stacked together. At the native resolution of ACIS ( 0 ". 492 pixels), images of the entire AEGIS-XD region would be too large to manipulate efficiently. We therefore created separate image stacks for the AEGIS-1, AEGIS-2, and AEGIS-3 fields. These overlap significantly, meaning that edge effects can be avoided, but in the final catalog we remove duplicated entries detected in more than one image (see below). Exposure maps for the stacked images were created by summing the exposure maps of 
Table 3

Weights Used for Exposure Map Calculations

\begin{tabular}{lcccc}
\hline \hline \multirow{2}{*}{$\begin{array}{l}\text { Energy } \\
(\mathrm{keV})\end{array}$} & Full & Soft & Hard & Ultrahard \\
\cline { 2 - 5 } & 0.2480 & 0.3867 & $\ldots$ & $\ldots$ \\
0.65 & 0.1509 & 0.2352 & $\ldots$ & $\ldots$ \\
0.95 & 0.1042 & 0.1625 & $\ldots$ & $\ldots$ \\
1.25 & 0.0776 & 0.1209 & $\ldots$ & $\ldots$ \\
1.85 & 0.0607 & 0.0947 & $\ldots$ & $\ldots$ \\
2.50 & 0.1359 & $\ldots$ & 0.3789 & $\ldots$ \\
3.50 & 0.0842 & $\ldots$ & 0.2346 & $\ldots$ \\
4.50 & 0.0590 & $\ldots$ & 0.1645 & 0.4256 \\
5.50 & 0.0445 & $\ldots$ & 0.1240 & 0.3208 \\
6.50 & 0.0352 & $\ldots$ & 0.0980 & 0.2536 \\
\hline
\end{tabular}

the individual ObsIDs. Figure 2 shows the effective exposure time as a function of survey area for the AEGIS-XD data.

\subsection{Source Detection}

Source detection was the same as described in L09 and the reader is referred to that paper for full details. Briefly, a "seed" source catalog was first created using the wavdetect task run at a low probability threshold $\left(10^{-4}\right)$ on the mosaic images. This low threshold is intended to capture all potential sources, but likely contains many spurious sources. Aside from this thresholding, the only information used from wavdetect in the final catalog is the source position. Using these positions for the candidate sources, counts were extracted from the mosaic images using a circular aperture with radius equal to the exposure-weighted $70 \%$ encircled energy fraction (EEF) of the Chandra point-spread function (PSF). The PSFs were taken from a lookup table calculated using the MARX simulation software as described in L09. Background was determined using an annulus with inner radius equal to 1.5 times the $95 \%$ EEF at the source position and outer radius 100 pixels larger than this, excluding detected sources. The background was then scaled to the size of the source region, and the Poisson false probability of observing the total counts given the expected background was calculated, masking out the 95\% EEF of candidate sources. A significance threshold of $4 \times 10^{-6}$ was then applied, and a further detection iteration was performed, masking out only the sources more significant than this. This iteration ensures that the background is not underestimated due to the masking of random positive variations identified as candidate sources in the wavdetect seed list. Any source detected at this $4 \times 10^{-6}$ probability level in the second iteration in any individual band was included in the final catalog. The sources that were considered significant were band-merged using the matching criteria specified in Table 2 of L09. Photometry was then performed to estimate the fluxes in several energy ranges, even if the source was not considered a significant detection in that particular band. After performing the band merging, we visually inspected the images of the sources in each of the fields and checked that the correct crossband counterparts were identified. Two sources in the catalog were detected in the soft and full bands, but not correctly matched with their hard (and in one case ultrahard) band counterparts. These were combined manually. Two single-band detected sources (one soft, one ultrahard) were removed from the catalog after visual inspection revealed strong contamination from nearby bright sources. These removed sources were

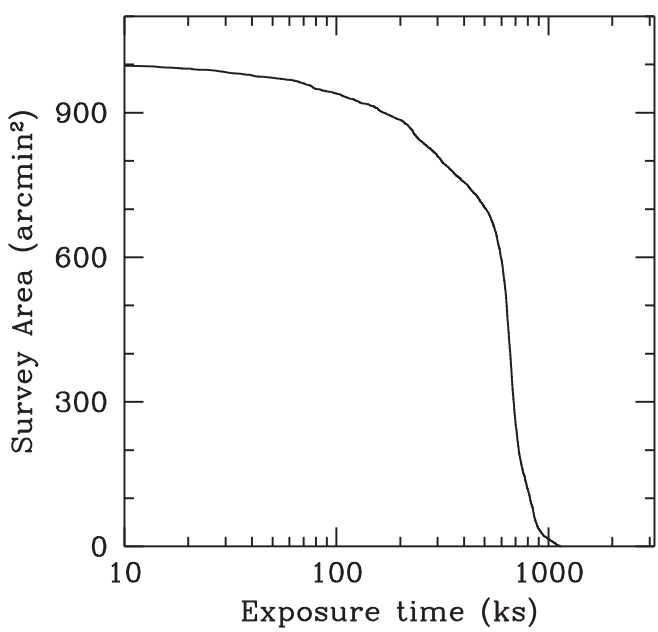

Figure 2. Effective exposure time as a function of survey area for the AEGISXD observations.

most likely incorrectly identified in the initial wavdetect seed catalog.

Finally, the source catalogs for the individual subfields AEGIS 1-3 were merged to remove duplicate sources in the overlapping regions. A $2^{\prime \prime}$ search radius was adopted, and as in L09, the source was chosen from the field with the smallest offaxis angle for the final catalog.

One significant change in the current paper compared to L09 is in the X-ray photometry. Specifically, here we have adopted elliptical apertures to extract the counts from the individual ObsIDs using the 90\% EEF PSF appropriate for the ObsID in question (95\% in the case of the soft band). This contrasts with the source detection described above, and the photometry in L09, both of which adopt circular apertures. Fluxes were estimated using the Bayesian methodology described in L09 using a $\Gamma=1.4$ spectrum with Galactic $N_{\mathrm{H}}$ of $1.3 \times 10^{20} \mathrm{~cm}^{-2}$ (Dickey \& Lockman 1990). The count rates in the full, hard, and ultrahard bands were also extrapolated to fluxes in standard energy bands: $0.5-10,2-10$, and $5-10 \mathrm{keV}$, respectively. Hardness ratios were calculated using the Bayesian methodology BEHR (Park et al. 2006).

\subsection{Spitzer/IRAC Observations}

The IRAC Guaranteed Time Observations of the AEGIS field cover a region approximately $2^{\circ}$ by $10^{\prime}$ (Barmby et al. 2006, 2008). The ACIS field of view is wider than this (see Figure 3), meaning that the GTO IRAC observations miss the edges of the deep Chandra imaging. As shown by L09, for example, and discussed below, Spitzer IRAC observations are critical for secure identifications of X-ray sources. For this reason, as part of the Chandra program we obtained additional Spitzer IRAC imaging of the edges of the strip. The IRAC coverage map is shown in Figure 1, and the data reduction was performed as described in Barro et al. (2011a), and incorporated into the Rainbow database.

\section{POINT SOURCE CATALOG}

The final point source catalog in the AEGIS-X Deep area consists of 937 sources. Of these 859, 732, 574, and 299 , respectively, were detected at $p<4 \times 10^{-6}$ in the full, soft, hard, and ultrahard bands. Sources detected in one band but not another are detailed in Table 4. The AEGIS-XD X-ray 


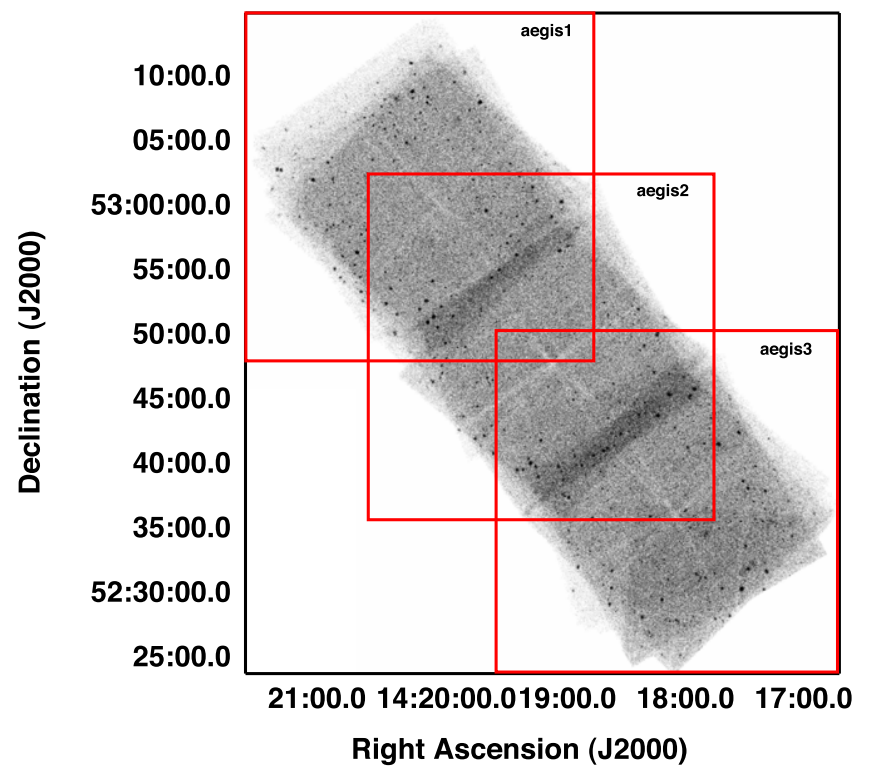

Figure 3. Mosaic full band image of the Extended Groth Strip showing the location and overlap of the three central AEGIS-XD fields, which have nominal $800 \mathrm{ks}$ depth. The subfields AEGIS-1, AEGIS-2, and AEGIS-3 are shown as red squares.

source catalog with full X-ray information is made publicly available in FITS table format, as detailed in an Appendix to this paper and at http://www.mpe.mpg.de/XraySurveys/. As a demonstration of the properties of the AEGIS-XD X-ray soruces, Figure 4 presents the hardness ratio distribution of the hard-band (2-7 KeV) selected sample. This is compared to the hardness ratio of hard-band detected sources in the AEGIS-XW (L09) and the 4Ms CDFS (Rangel et al. 2013). These hardness ratios were estimated from the counts in the $0.5-2$ and $2-7 \mathrm{keV}$ spectral bands.

\subsection{Sensitivity}

Sensitivity maps were computed according to the procedure described in Georgakakis et al. (2008b) as implemented by L09. This approach accounts for incompleteness and Eddington bias in the sensitivity calculation, which is performed in a manner that is also fully consistent with the source detection procedure. The flux limits for the new AEGIS-XD survey as a function of area are shown for various energy bands in Figure 5, compared to the deepest X-ray survey in existence, the Chandra Deep Field South, together with the sensitivity curves of the G12 source catalog of the entire EGS Chandra survey. Following L09, we define the limiting flux of our observations as the flux to which at least $1 \%$ of the survey area is sensitive. We find the limiting fluxes so defined to be $1.5 \times 10^{-16}(\mathrm{FB} ; 0.5-10 \mathrm{keV}), 3.3 \times 10^{-17}(\mathrm{SB} ; 0.5-2 \mathrm{keV})$, $2.5 \times 10^{-16}(\mathrm{HB} ; 2-10 \mathrm{keV})$, and $3.2 \times 10^{-16} \mathrm{erg} \mathrm{cm}^{-2} \mathrm{~s}^{-1}$ (UB; $5-10 \mathrm{keV}$ ). We also show in Table 5 the $50 \%$ and $90 \%$ completeness limits of the survey.

\subsection{False Source Estimation}

The source detection algorithm applied here (and in L09) is designed to provide an accurate estimate of the sensitivity of the X-ray observations at each position. The detection threshold can be altered depending on the number of likely spurious detections to be deemed acceptable in the catalog. Here we
Table 4

Sources Detected in One Band but Not Another

\begin{tabular}{lccccc}
\hline \hline Detection & Total Number & \multicolumn{4}{c}{ Non-detection Band } \\
\cline { 3 - 6 } Band $(\mathrm{keV})$ & of Sources & Full & Soft & Hard & Ultrahard \\
\hline Full $(0.5-7)$ & 859 & $\ldots$ & 190 & 299 & 562 \\
Soft $(0.5-2)$ & 732 & 63 & $\ldots$ & 282 & 478 \\
Hard $(2-7)$ & 574 & 14 & 124 & $\ldots$ & 277 \\
Ultrahard $(4-7)$ & 299 & 2 & 45 & 2 & $\ldots$ \\
\hline
\end{tabular}

adopt a relatively conservative threshold, which should result in only a small number of spurious X-ray detections; L09, for example, estimated that $<1.5 \%$ of the AEGIS-XW $200 \mathrm{ks}$ sources were likely to be spurious. To assess this for the deeper AEGIS-XD data, we performed tests of the source detection on simulated X-ray fields. The number of spurious sources expected in any given field may depend on the exposure map of the field: for example, with multiple overlapping pointings, as are present here, edge effects might introduce problems into the source detection.

The false source content of our catalog was estimated based on the field configuration of the AEGIS-2 field, overlapping the individual subframes just as in the real data. The simulations were initially run just using the background, subsequently applying the source detection procedure for the real observations to these simulated background-only images for the real observations. Correcting the area of the simulated observations to the total AEGIS-XD survey, we predict about 12 spurious sources in the catalog (i.e., around $1.3 \%$ ), which is consistent with the estimates from L09. We also estimated the false source contamination using simulated sources, based on the $\log \mathrm{N}-$ $\log S$ function of Georgakakis et al. (2008b), yielding similar results.

A further estimate of the spurious source content can be made using the ultrahard band (UB) images. Due to a combination of the strong energy-dependence of the effective area and PSF of the Chandra optics (which is inherent in the Wolter-1 design) and the nature of the spectra of the underlying source population, it is unlikely for real sources to be detected in only the UB, because it is the least sensitive of all the detection bands. As a result, UB-only sources must have heavy obscuration at just the right level to suppress the soft, full, and hard band detections below the threshold, but not so high that it also suppresses the UB flux. Furthermore, in practice, even heavily obscured sources are often detected in the soft X-ray band due to the presence of scattered X-ray light (e.g., Brightman \& Nandra 2012). None of these considerations applies to spurious sources, which, if present in the ultrahard band, should not be detected in any other band. One source in our catalog was detected only in the UB, and is thus a candidate for being spurious in the UB catalog, which contains a total of 299 sources. This consideration suggests that the simulations might overestimate the number of spurious sources in our catalog.

With our deeper X-ray data, we can also make a post-hoc check of the number of spurious sources in the $200 \mathrm{ks}$ L09 catalog. Naively, it would be expected that all real sources in the AEGIS-XW catalog also appear in our deeper observations in the overlapping area. This is not the case, however, if the sources are spurious because the significance would tend to go down (and eventually below the detection threshold) with deeper data. There are 17 sources in the AEGIS-XW catalog, 

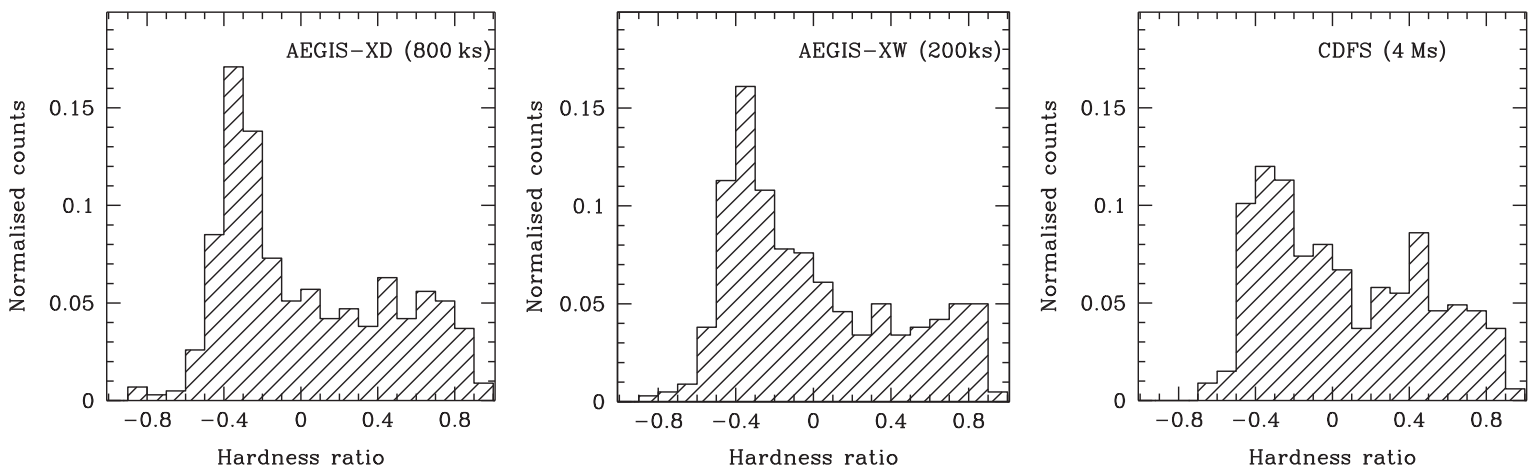

Figure 4. Hardness ratio distribution of hard-band (2-7 keV) selected sources in the AEGIS-XD (left panel; this paper), AEGIS-XW (middle panel; L09), and 4Ms CDFS (right panel; Rangel et al. 2013). The hardness ratio is determined from the counts in the $0.5-2$ and $2-7 \mathrm{keV}$ bands.
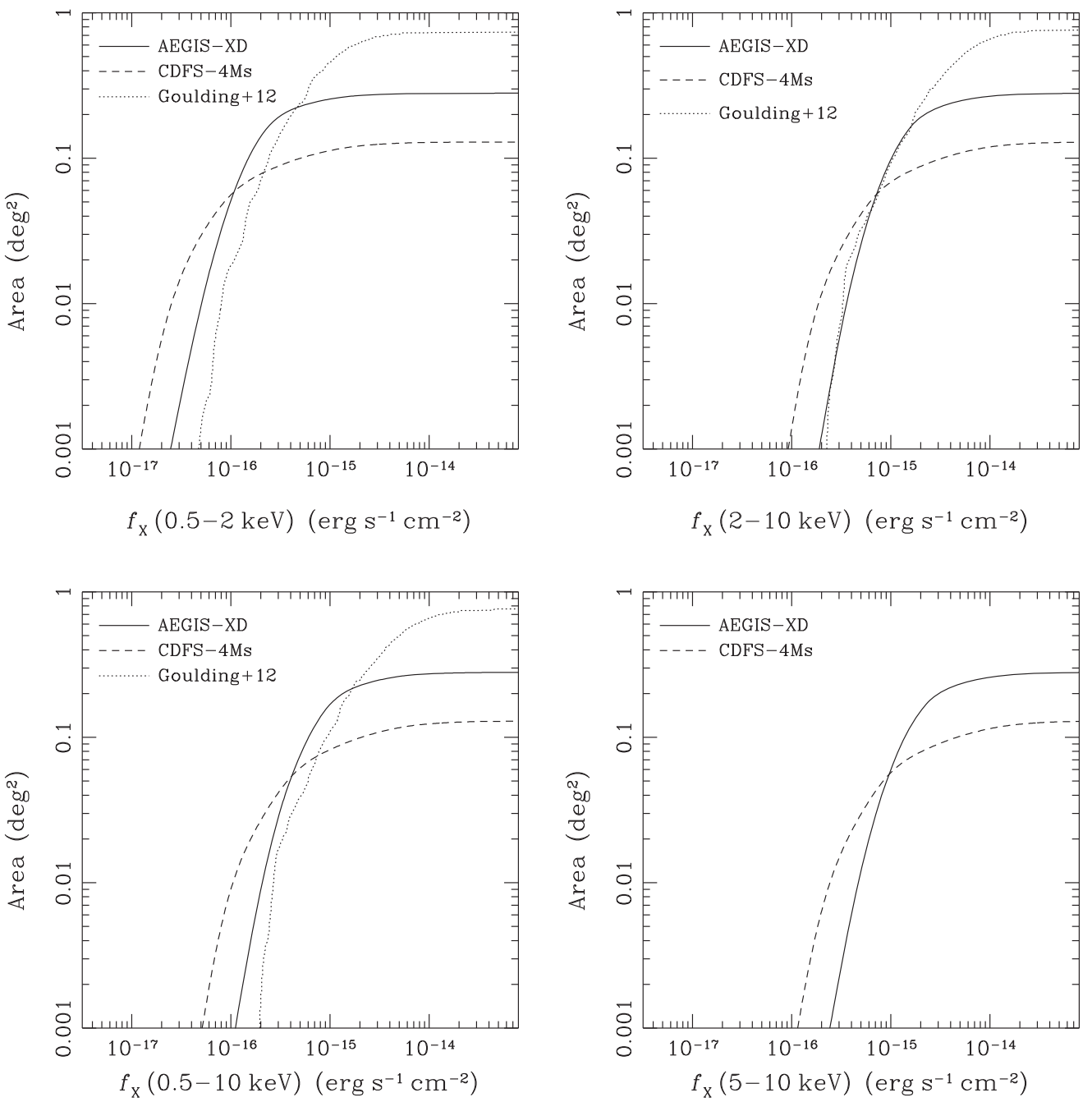

Figure 5. Sensitivity curves for the AEGIS-XD survey in the soft, hard, full, and ultrahard bands (solid lines), calculated using the methodology of Georgakakis et al. (2008b). These are compared to the 4Ms Chandra Deep Field South (dashed lines). The 4Ms CDFS reaches the deepest limits of any X-ray survey, but the AEGISXD data provide a considerable increase in area. Also shown in the plot are the sensitivity curves of the G12 in the entire Extended Groth Strip Chandra survey (deep and wide). For this comparison, we apply conversions to account for the different power-law X-ray spectral index assumed by G12 to determine the fluxes and different energy bands used in their work compared to this paper. Note, however, that their sensitivity calculation follows a different methodology, as does their calculation of the exposure maps.

which are covered in the deeper Chandra pointings but not listed as significant sources in our AEGIS-XD catalog. These are listed in Table 6, with postage-stamp images of the objects shown in Figure 6. The images show that the majority of the cases are low significance detections in the $200 \mathrm{ks}$ catalog that are not confirmed in the deeper data. Two (egs 0511 and egs_0529) are sources that were detected in the wings of nearby bright (and possibly extended) sources. A relatively large number of the others are in the very central regions of the image where the PSF is small, and where the original 
Table 5

X-Ray Flux Completeness Limits for the AEGIS-XD Survey

\begin{tabular}{llcc}
\hline \hline & \multicolumn{3}{c}{ Completeness Limit $^{\mathrm{a}}$} \\
\cline { 2 - 4 } Band & $1 \%^{\mathrm{b}}$ & $50 \%^{\mathrm{b}}$ & $90 \%^{\mathrm{b}}$ \\
\hline Full & 1.46 & 8.22 & 35.9 \\
Soft & 0.33 & 2.02 & 9.22 \\
Hard & 2.48 & 13.6 & 58.2 \\
Ultrahard & 3.27 & 18.4 & 82.2 \\
\hline
\end{tabular}

Notes.

${ }^{a}$ Flux to which $1 \%, 50 \%$ and $90 \%$ of the survey area is complete.

${ }^{\mathrm{b}}$ Fluxes are in units of $10^{-16} \mathrm{erg} \mathrm{cm}^{-2} \mathrm{~s}^{-1}$.

detections by L09 are based only on a very small number of photons. In these and indeed other cases the objects identified by L09 may not be truly spurious but simply represent sources whose true flux lies below the detection limit even of the $800 \mathrm{ks}$ data, but which generated a significant number of counts in the 200 ks observation due to Poisson statistics. In other words, they may be sources that were originally detected due to the Eddington bias. This assertion is supported by the fact that many have optical counterparts (see Table 6). This may also be explained if the X-rays are variable and the true flux has dropped by a large factor since the initial observations by L09.

\subsection{Comparison with Goulding et al. (2012)}

G12 have already published a catalog of X-ray sources and optical associations in this field. The set of Chandra data used in their catalog is similar but not identical to ours, in that they analyze the entire AEGIS-X area, both deep and wide, whereas we use only the regions with nominal 800 ks exposure. Further, we use two more ObsIDs in these areas, 9876 and 9881, which are not analyzed by G12. The other 55 of our pointings are in common with G12, who nonetheless adopt a different procedure for data reduction, source detection, and identification of the X-ray sources. A comparison of the last can be found in Section 4.2.

We cross-correlated the X-ray source list of G12 with our catalog using a match radius of $3^{\prime \prime}$, and restricting to the common area. From this we find 115 sources in G12 that have no match in our catalog. The vast majority (108) are faint sources close to the detection threshold (see Figure 7). In these cases, small differences in the analysis or source detection procedure (in particular the adopted detection threshold) can easily account for a detection in one catalog but not the other. In the photon-starved regime of Chandra the inclusion or exclusion of a single count in the source detection cell can easily change a "detection" to a "non-detection" or vice versa. Relatively minor differences in the determination of the background can have a similar effect. The other seven sources in G12 but not in our catalog are brighter and potentially a greater cause for concern. Visual examination shows three that are far off axis and have a counterpart in our catalog, but just outside the $3^{\prime \prime}$ match radius. The remaining four are in crowded or bright source regions. Visual inspection suggests that these may indeed be distinct sources, but have not been separated or identified as such by our detection algorithm.

Performing the opposite comparison, we find 73 sources in our catalog that have no counterpart within $3^{\prime \prime}$ in G12. An examination of Figure 7 shows that a number of these sources are rather X-ray bright $\left(F_{0.5-10}>10^{-15} \mathrm{erg} \mathrm{cm}^{-2} \mathrm{~s}^{-1}\right)$. Examining the spatial distribution of these sources in our images, we find that the majority are at large off-axis angles, where the PSF is relatively broad.

We also compared the positions of our X-ray sources with those of G12, with the results of the comparison being shown in Figure 8. A systematic offset is found amounting to 0". 37 . This may be attributed in part to the different astrometric solutions adopted for the $\mathrm{X}$-ray images, with ours being tied to the CFHTLS $i$-band, and the G12 positions registered to the DEEP2 reference frame.

\section{MULTIWAVELENGTH COUNTERPARTS AND PHOTOMETRY}

We have identified multiwavelength counterparts to sources in our merged X-ray catalog using the likelihood ratio method (Ciliegi et al. 2003; Brusa et al. 2007; L09; Luo et al. 2010) and photometry from the Rainbow Cosmological Surveys Database $^{19}$ (Barro et al. 2011a, 2011b). The Rainbow database is a compilation of the photometric datasets in several of the deepest extragalactic fields, including the AEGIS field. Table 7 lists the relevant datasets. The multiwavelength images were registered to a common astrometric reference frame, and photometry was performed in consistent apertures to produce spectral energy distributions that span from the UV to mid-IR.

To identify our X-ray sources, we first searched for counterparts in any of the multiwavelength images (based on SExtractor catalogs generated from each of the images) within 3 3.5 of the X-ray position. All the possible counterparts were then cross-matched to each other using a $2^{\prime \prime}$ search radius to create a single multiband catalog. Next, we performed photometry using the same elliptical (Kron) aperture across all the optical and NIR bands. The aperture was defined in a reference image for each source, typically the deepest available ground-based optical image. If the source had a Kron radius $<4$."5 then we extracted IRAC photometry using a $2^{\prime \prime}$ circular aperture and applied standard aperture corrections, thus accounting for the larger PSF of the IRAC images. If the source was detected in IRAC only, we applied a $1^{\prime \prime} .5$ aperture in the optical/NIR images and forced a photometric measurement. If a single IRAC source was associated with multiple optical/NIR counterparts, the positions of the optical/NIR sources were adopted and used to deblend the IRAC photometry. The full procedure is described in Barro et al. (2011a).

We note that our procedure does not require a significant detection in IRAC or any specific optical/NIR band, and instead identifies potential counterparts to the X-ray sources in any available image (cf. the catalog presented by Barro et al. 2011a where an IRAC 3.6 or $4.5 \mu \mathrm{m}$ detection was required).

All of our X-ray sources (within the Rainbow coverage) have at least one candidate counterpart within the 3 ".5 search radius, with $\sim 58 \%$ having two or more.

In the next step we applied the likelihood ratio technique to determine which of these candidates is likely to be the true counterpart to the X-ray source, as opposed to a chance alignment. We first restricted the list of candidates to those with significant detections in a single, given optical, NIR, or mid-IR band with a measured magnitude, $m$. The likelihood ratio

\footnotetext{
${ }^{19}$ http://rainbowx.fis.ucm.es/Rainbow_Database/Home.html
} 
Table 6

AEGIS-XW Sources from L09 Not Included in AEGIS-XD Catalog

\begin{tabular}{|c|c|c|c|c|c|c|c|}
\hline $\begin{array}{l}\text { L09 } \\
\text { cat ID }\end{array}$ & $\begin{array}{l}\text { R.A. J2000 } \\
\quad(\mathbf{J} 2000)\end{array}$ & $\begin{array}{l}\text { Decl. J2000 } \\
(\mathrm{J} 2000)\end{array}$ & $p_{\min }{ }^{\mathrm{a}, \mathrm{b}}$ & $\begin{array}{l}\text { Det. }^{\text {a }} \\
\text { Bands }\end{array}$ & $\begin{array}{c}\text { Bayesian flux }{ }^{\mathrm{a}, \mathrm{c}} \\
\left(10^{-16} \mathrm{erg} \mathrm{s}^{-1} \mathrm{~cm}^{-2}\right)\end{array}$ & $\begin{array}{l}i_{\mathrm{AB}}^{\prime}{ }^{\mathrm{d}} \\
(\mathrm{mag})\end{array}$ & $\begin{array}{l}\text { Classical Flux Limit } \\
\left(10^{-16} \mathrm{erg} \mathrm{s}^{-1} \mathrm{~cm}^{-2}\right)\end{array}$ \\
\hline egs_0379 & 214.363649 & 52.525411 & $1.0 \times 10^{-8}$ & $f_{s}$ & $8.8_{-2.7}^{+3.2}$ & 25.12 & $<2.6$ \\
\hline egs_0463 & 214.491077 & 52.632192 & $3.6 \times 10^{-6}$ & $s$ & $<2.8$ & 18.43 & $<2.3$ \\
\hline egs_0503 & 214.530413 & 52.508574 & $3.3 \times 10^{-6}$ & $s$ & $<3.9$ & 21.78 & $<2.2$ \\
\hline egs_0511 & 214.548133 & 52.759199 & $1.0 \times 10^{-8}$ & $\mathrm{f}$ & $20.4_{-5.3}^{+5.9}$ & 23.69 & $<3.7$ \\
\hline egs_0521 & 214.559843 & 52.568176 & $2.1 \times 10^{-6}$ & $s$ & $<8.2$ & $>27.0$ & $<4.2$ \\
\hline egs_0528 & 214.572498 & 52.446772 & $3.2 \times 10^{-6}$ & $f$ & $5.8_{-4.2}^{+3.2}$ & 23.79 & $<4.9$ \\
\hline egs_0529 & 214.573633 & 52.731312 & $1.0 \times 10^{-8}$ & $f_{s}$ & $9.2_{-3.3}^{+3.9}$ & 24.89 & $<2.6$ \\
\hline egs_0533 & 214.575732 & 52.442809 & $3.6 \times 10^{-6}$ & $f$ & $4.3_{-2.8}^{+3.8}$ & 22.00 & $<4.9$ \\
\hline egs_0555 & 214.597394 & 52.488107 & $1.6 \times 10^{-6}$ & $s$ & $<1.5$ & 21.97 & $<7.1$ \\
\hline egs_0590 & 214.643180 & 52.698915 & $4.8 \times 10^{-7}$ & $f_{s}$ & $4.7_{-2.5}^{+3.1}$ & $>27.0$ & $<1.8$ \\
\hline egs_0593 & 214.653435 & 52.627987 & $4.8 \times 10^{-7}$ & $s$ & $3.6_{-1.9}^{+2.5}$ & 22.23 & $<1.4$ \\
\hline egs_0602 & 214.662429 & 52.684361 & $3.3 \times 10^{-6}$ & $s$ & $<2.9$ & 25.39 & $<1.8$ \\
\hline egs_0687 & 214.813366 & 52.856652 & $3.0 \times 10^{-6}$ & $h$ & $2.4_{-1.3}^{+1.9}$ & $>27.0$ & $<1.4$ \\
\hline egs_0688 & 214.815105 & 52.793307 & $2.7 \times 10^{-6}$ & $h$ & $2.4_{-1.7}^{+1.9}$ & $>27.0$ & $<1.6$ \\
\hline egs_0711 & 214.856439 & 52.745895 & $4.8 \times 10^{-7}$ & $s$ & $5.9_{-3.3}^{+3.5}$ & 24.28 & $<3.5$ \\
\hline egs_0728 & 214.877813 & 53.007428 & $1.0 \times 10^{-8}$ & $f_{s}$ & $1.6_{-0.7}^{+0.7}$ & 21.54 & $<4.6$ \\
\hline egs_0851 ${ }^{\mathrm{f}}$ & 215.076587 & 53.032611 & $2.6 \times 10^{-6}$ & $s$ & $3.2_{-1.7}^{+2.5}$ & 18.99 & $<1.4$ \\
\hline
\end{tabular}

Notes.

a Values from L09.

b Minimum false detection probability found for the four analysis bands.

c Full band flux or upper limit.

d Optical identification from L09 or upper limit where no counterpart exists.

e Values from this work.

${ }^{\mathrm{f}}$ Source also detected in G12.
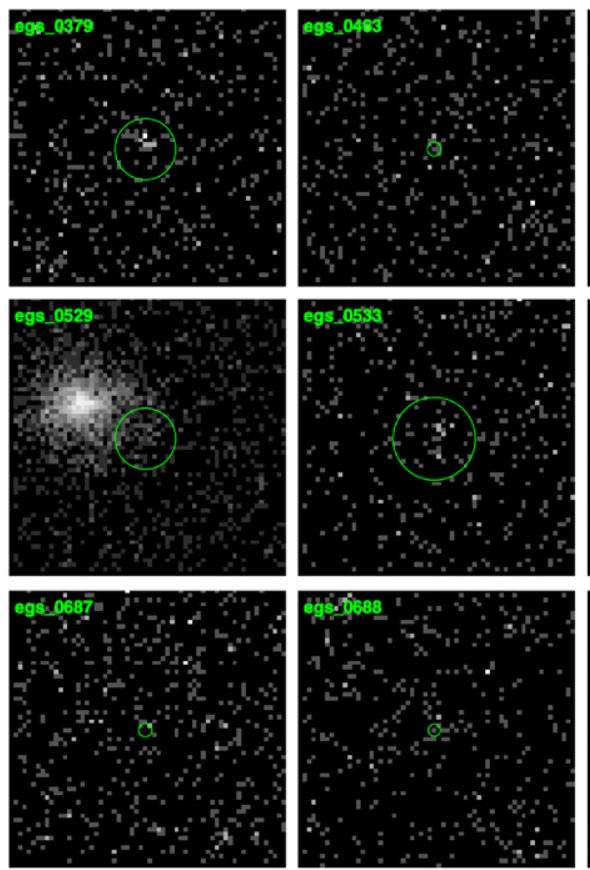
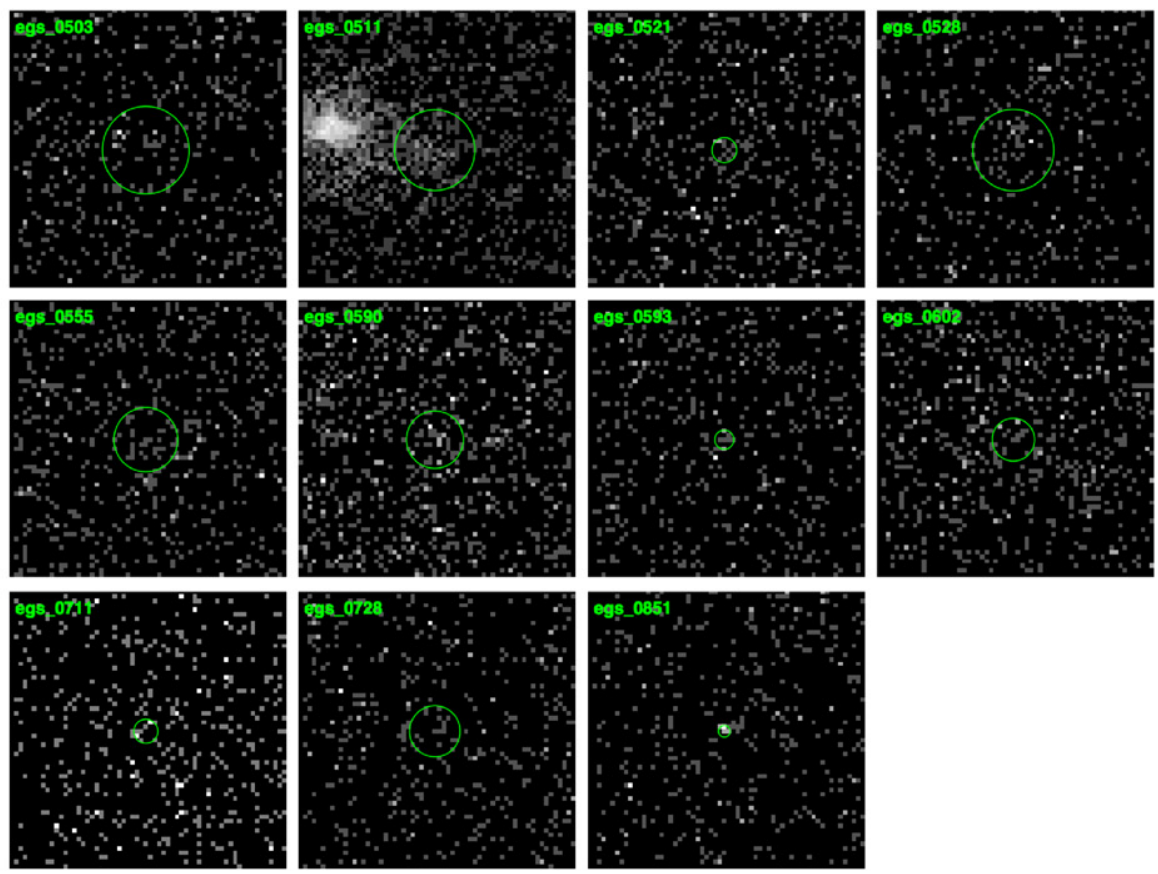

Figure 6. Sources in the $200 \mathrm{ks}$ AEGIS-XW catalog of L09 not significantly detected in the deeper $800 \mathrm{ks}$ data. In each case, the green circle shows the L09 position overlaid on the $800 \mathrm{ks}$ images from this work. Generally these are low significance detections that are not confirmed in the deeper data, but in a few cases the L09 detection appears in the wings of a nearby brighter source. The size of the circle is equal to the $90 \%$ EEF in the full band, while the cutouts have a size of $25 \times 25^{\prime \prime}$.

compares the probability that a candidate counterpart with magnitude $m$ found at a distance $r$ from the X-ray source position is the true counterpart and the probability that it is a spurious background source.
The likelihood ratio (LR) is given by

$$
\mathrm{LR}=\frac{q(m) f(r)}{n(m)}
$$




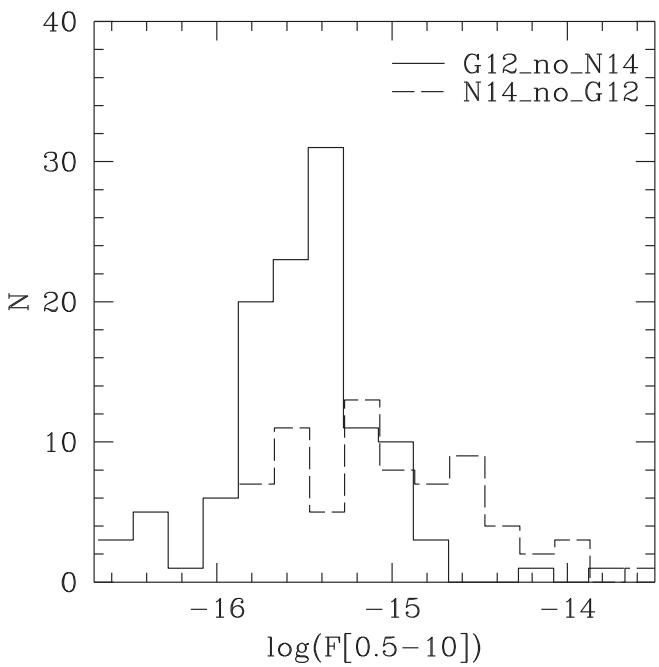

Figure 7. X-ray flux distribution of the sources that are in our catalog and not that of G12, or vice versa. G12 include a number of faint sources in their catalog that do not satisfy our false probability threshold. A number of bright sources are included in our catalog, but not in that of G12. These are generally at the edges of the field, where the PSF is relatively large.

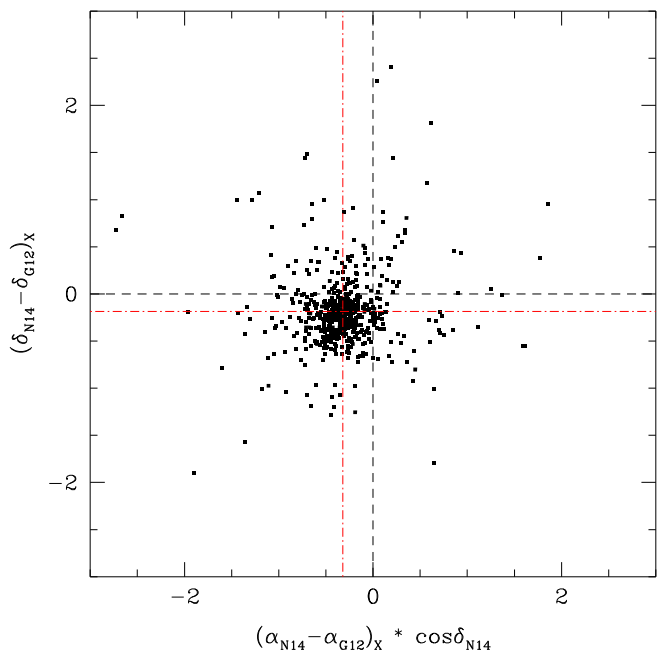

Figure 8. Offset between the X-ray positions of sources in our catalog, and those in G12, in cases where the source is matched within $3^{\prime \prime}$ radius. There is a systematic offset of around 0 ". 4 .

where $q(m)$ is the expected magnitude distribution of the true counterparts to the X-ray sources, $n(m)$ is the surface density of background sources as a function of magnitude, and $f(r)$ is the probability distribution of angular separations of the sources. We assume $f(r)$ can be described by a symmetric twodimensional Gaussian distribution,

$$
f(r)=\frac{1}{2 \pi \sigma^{2}} \exp \left(\frac{-r^{2}}{2 \sigma^{2}}\right)
$$

where the standard deviation, $\sigma$, combines the $\mathrm{X}$-ray and counterpart positional uncertainties, which were added in quadrature. The X-ray positional uncertainties that are used depend on the source counts and off-axis angle, as described in L09. Because our Rainbow counterpart catalog was limited to sources within $3^{\prime \prime} .5$ of an X-ray source, we estimated the background source density, $n(m)$, using the original SExtractor catalogs for the given band over the entire field, restricting to sources with significant detections and measured magnitudes, $m$, in that band. This approach provides an accurate estimate of the background source density using the entire photometric coverage.

The magnitude distribution of true counterparts, $q(m)$, was estimated via the iterative method described in Luo et al. (2010). Briefly, a first estimate of $q(m)$ was determined by matching counterparts to X-ray sources within a small radius (we adopted 1".5), and subtracting the background density. The LR was then calculated for all counterparts to X-ray sources within our 3 ".5 search radius. We found the counterpart with the highest LR value for each X-ray source and applied a threshold, $\mathrm{LR}_{\text {thresh }}$, that maximizes the sum of the sample completeness and reliability to identify a sample of "secure" matches. These secure matches were then used for a new estimate of $q(m)$, and the likelihood ratios recalculated. This process was repeated 10 times, resulting in a stable number of matches and threshold $L R$ values.

We repeated the entire LR matching process for the bands indicated in column 3 of Table 7. Finally, we combined the matches to produce a master list of counterparts. We first took the secure counterparts in the highest priority band (indicated by LR priority=1). Next, we looped through the remaining match bands and assigned a final counterpart if a secure match was available in that band (and not available in any of the higher priority bands). No additional cross-matching is required to obtain the full multiband photometry as this is provided through matched apertures for all the Rainbow counterparts, regardless of the detection band (c.f. Luo et al. 2010). In practice, the vast majority $(\sim 90 \%)$ of X-ray sources are assigned final counterparts from the highest priority match band, IRAC $3.6 \mu \mathrm{m}$. IRAC is known to give the highest match rate for faint X-ray sources in deep Chandra surveys (Cardamone et al. 2008; L09). The additional steps help us identify counterparts when the IRAC candidate is faint, blended, or non-existent. We assigned a match to 929 of the 937 sources.

The X-ray fluxes in the full, soft, and hard bands are plotted against the counterpart magnitude in various matching bands in Figure 9. The lines of constant $F_{X} / F_{\text {opt }}$ show a clear effect that the counterparts are generally brighter in the Subaru MOIRCS NIR $\left(K_{s}\right)$ and IRAC $3.6 \mu \mathrm{m}$ bands than they are in the optical bands (CFHT $i^{\prime}$ or Subaru $R_{c}$ ). This illustrates the well-known fact that the X-ray sources-which are dominated by AGNreside in relatively red host galaxies (Barger et al. 2003; Nandra et al. 2007; Brusa et al. 2009; Civano et al. 2012), a fact which accounts for the much higher IRAC identification rate, compared with optical photometry. The figure also identifies objects that are newly detected in the 800 ks survey, as opposed to those which were already in the $200 \mathrm{ks}$ catalog of L09. While the fainter $800 \mathrm{ks} \mathrm{X}$-ray sources are typically identified with fainter optical counterparts, a small but significant fraction of the faintest $\mathrm{X}$-ray sources are identified with very bright optical sources $\left(R_{\mathrm{AB}}=20\right.$ or brighter). Most of these sources are secure stars as classified via spectroscopy, multiple color-color selection (see Barro et al. 2011a, 2011b), or spectral energy distribution (SED) fitting. Some of these sources are galaxies with a low X-ray luminosity, indicating that their X-ray emission may be dominated by stellar processes (e.g., via X-ray binaries and diffuse hot gas), rather than an accreting black hole. Looking more specifically at this 
Table 7

Multiwavelength Photometric Datasets Included in the Rainbow Database in Our Fields

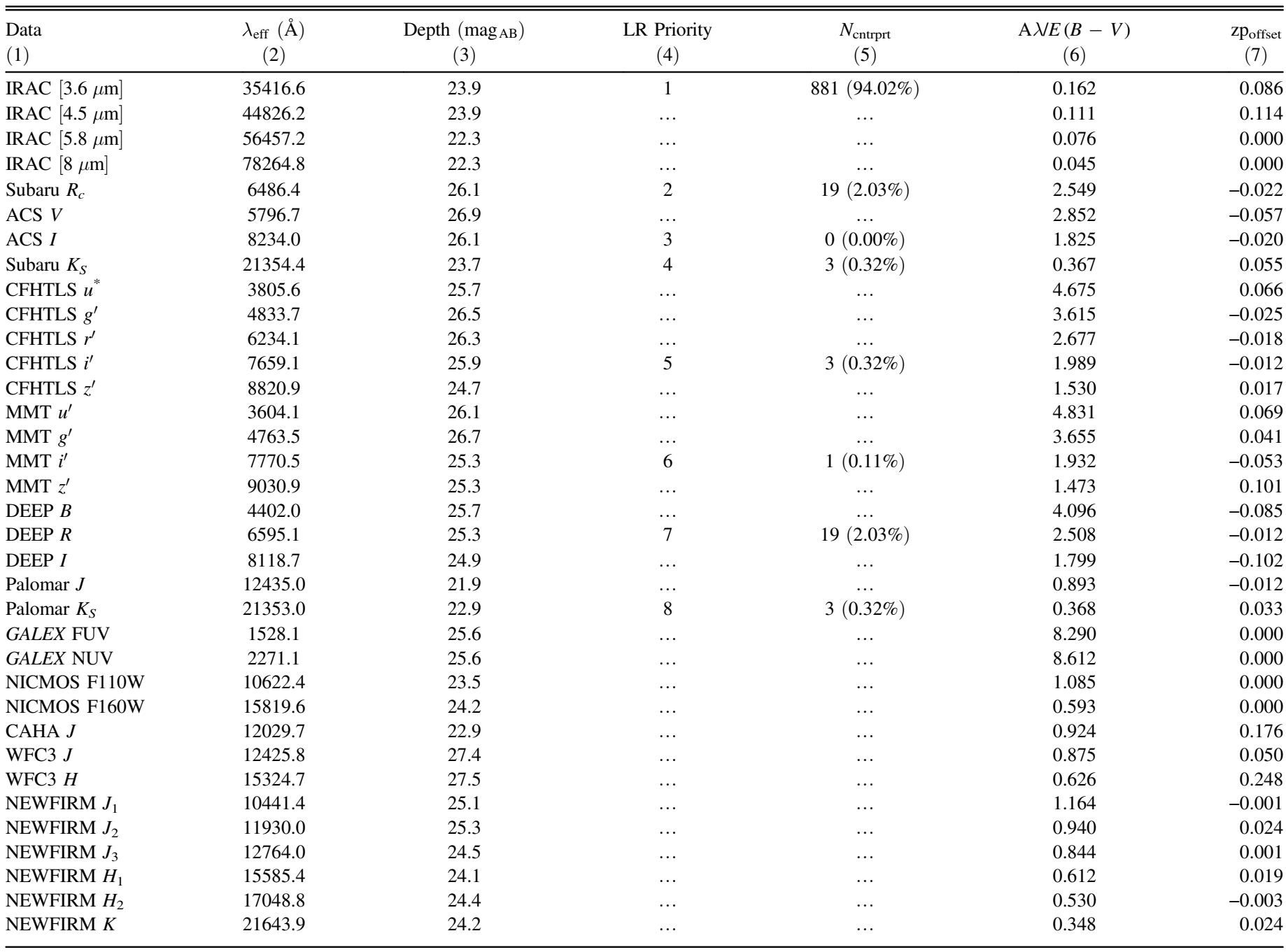

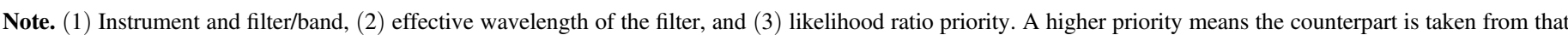

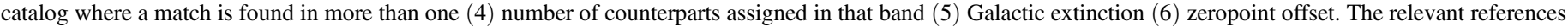

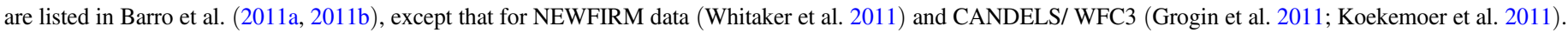

issue, we find a total of 49 sources with $F_{\mathrm{X}} / F_{\text {opt }}<-2-$ where $F_{\mathrm{X}}$ is the soft band flux and $F_{\mathrm{opt}}$ is based on the Subaru $R$ band-which are not flagged as stars. Of these, 44 have a secure spectroscopic redshift $\left(z_{\text {spec }}>0\right)$, while the others have reliable photo- $z>0$. All but one of these sources has $\log L_{\mathrm{X}}<42$, meaning that in principle they could be normal galaxies rather than AGN. They represent $6.7 \%$ of the soft $\mathrm{X}$-ray detected sample. This is consistent with the work of Lehmer et al. (2012), from which we would predict a normal galaxy fraction of $6.1 \%$ at $F_{\mathrm{X}}>1.96 \times 10^{-16} \mathrm{erg} \mathrm{cm}^{-2} \mathrm{~s}^{-1}$, which is our $1 \%$ completeness soft flux limit. We nonetheless caution that separating AGN and normal galaxies based on these criteria is difficult, and requires detailed consideration of the properties of the individual objects.

There is also some evidence from this figure to suggest that the fainter X-ray sources do not become significantly fainter in the longer wavelength NIR or IRAC bands. The IRAC $3.6 \mu \mathrm{m}$ magnitude, for example, remains relatively constant over the full range of X-ray fluxes probed by the AEGIS-XD survey. The interpretation of this is not straightforward, but may be related to the fact that X-ray selection tends to identify the most massive galaxies at any given redshift (e.g., Bundy et al. 2008; Aird et al. 2012), and/or that the optical faintness of many of the X-ray sources is due to dust reddening.

\subsection{Astrometric Accuracy}

Following the cross-matching procedure, we can make a post-hoc estimate of the astrometric accuracy of the X-ray positions in our catalog. Figure 10 shows the offsets between the X-ray position and that of the multiband counterpart. Overall, we find that $84 \%$ of the of the counterparts lie within $1^{\prime \prime}$ of the X-ray position, and $97 \%$ within $2^{\prime \prime}$. As discussed by L09, the astrometric accuracy is a function of both off-axis position and source counts (see their Figure 7 and Table 8). Figure 10 demonstrates the latter effect, whereby the positions degrade somewhat for fainter sources. For relatively bright $\mathrm{X}$-ray sources ( $>100$ net counts) we find $92 \%$ of counterparts within $1^{\prime \prime}$ and $99 \%$ within $2^{\prime \prime}$. With $<100$ net counts, we find $78 \%$ of the counterparts within $1^{\prime \prime}$, but still $96 \%$ within $2^{\prime \prime}$. The 


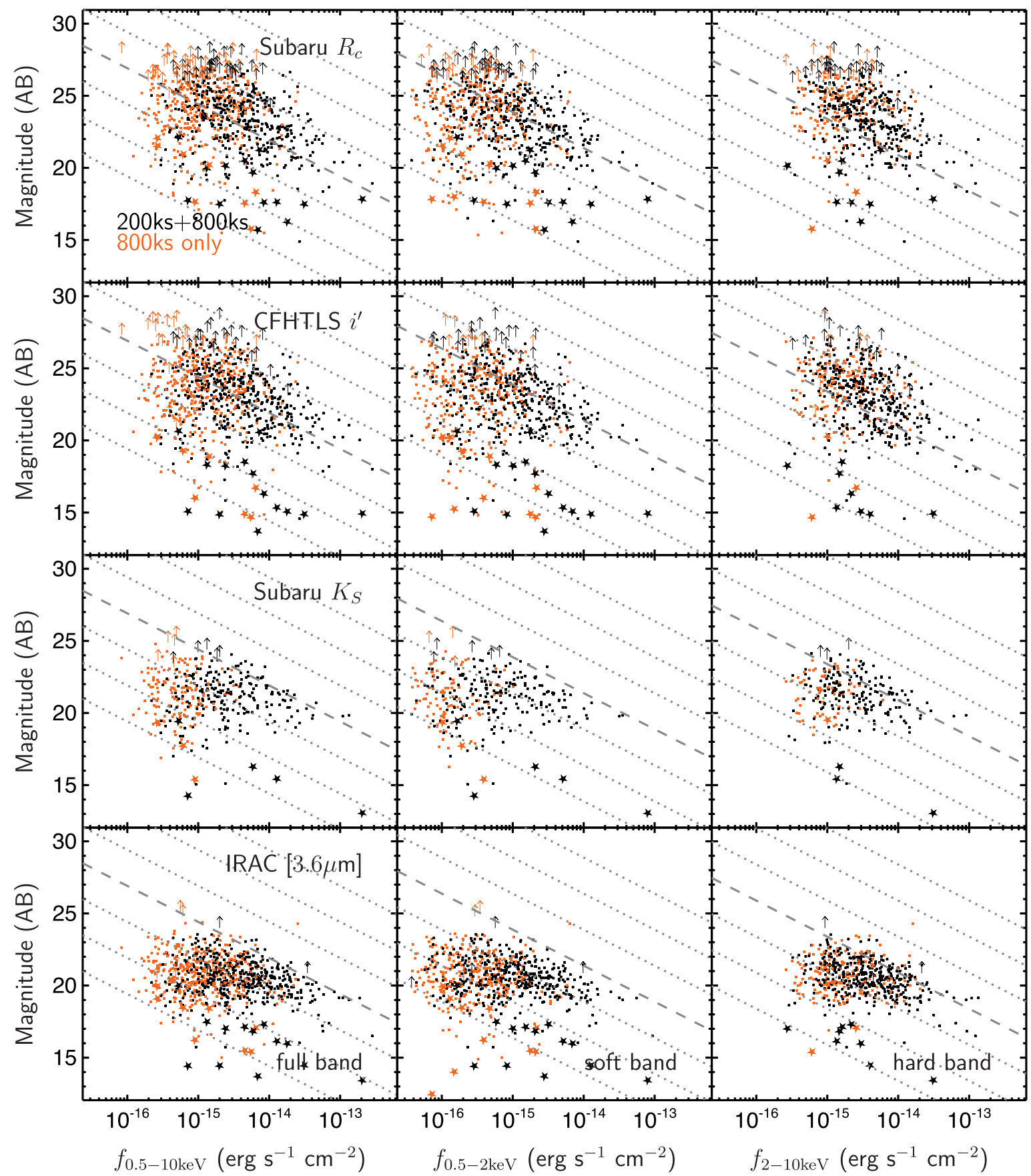

Figure 9. X-ray flux vs. AB magnitude in various matching bands for all AEGIS-XD 800 ks sources (orange symbols), and those also identified earlier in the shallower AEGIS-XW $200 \mathrm{ks}$ survey (black symbols). From top to bottom the comparison bands are the Subaru $R_{c}$-band, CFHTLS $i^{\prime}$, Subaru $K_{s}$, and IRAC $3.6 \mu$ m. Anything with less than $3 \sigma$ detection is shown as a limit (upward arrow) and stars are indicated with a star symbol. Note that the $K_{s}$ imaging covers a significantly smaller area than the other bands, accounting for the smaller number of matched sources. Lines denoting a constant ratio of the X-ray flux to the flux in the corresponding optical/IR band are shown as the dotted lines, with the dashed line representing a ratio of 1:1.

rms error for all sources is 0 ". $83\left(0^{\prime \prime} .62\right.$ for $>100$ net counts and 0 ". 93 for $<100$ counts).

\subsection{Comparison of Associations With G12}

For the $864 \mathrm{X}$-ray sources that are in common between our catalog and that of G12, we compared the counterpart identifications. There is a substantial difference in the multiwavelength datasets used: G12 used only the DEEP2 optical photometry, whereas we employ a much wider variety of data including (deblended) IRAC photometry, which is known to yield more efficient counterpart identification for X-ray sources (Cardamone et al. 2008; L09). The AGN are bright in the IRAC band and the number of background sources decreases, making it easier to obtain a secure association. Of the 864 common sources, G12 assign a counterpart to 606. By comparison, our method yields reliable associations for 830 sources, including 595 of the G12 counterparts. Of the $606 \mathrm{G} 12$ counterparts, our methodology indicates an unreliable association in 11 cases. 


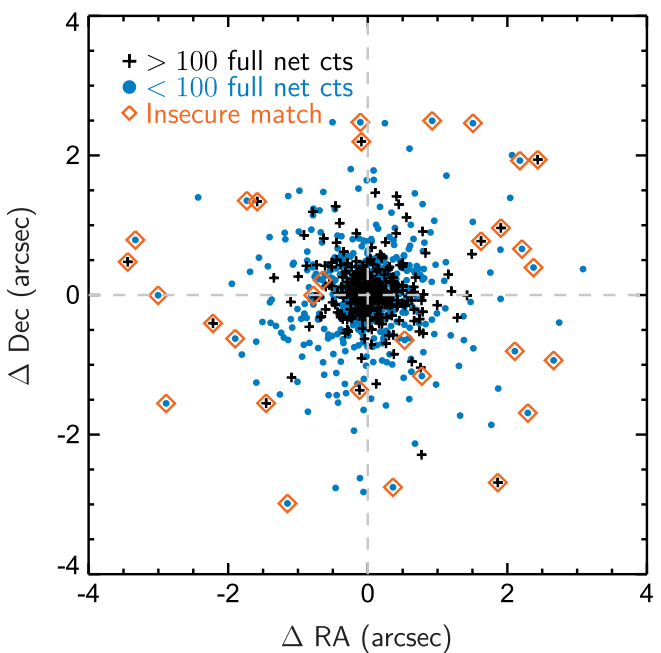

Figure 10. Offset between the X-ray and counterpart positions for the AEGIS$\mathrm{XD}$ sources. Black crosses show bright objects where the X-ray position is statistically well determined. With $<100$ net counts the positional accuracies degrade somewhat. However, the vast majority of the secure counterparts $(>97$ $\%$ ) lie within $2^{\prime \prime}$ of the X-ray position.

Matching the counterpart positions, we found a systematic offset between the counterpart positions of about 0 ".58 (see Figure 11). This is presumably due to the different astrometric systems adopted by DEEP2 and the Rainbow database. After correction for this offset, we found 572 of the 595 sources with high confidence counterparts in both works to be within $0^{\prime \prime} .5$ of each other, and hence are presumably the same counterpart (see Figure 11). For the remaining 23 sources we seem to identify a different counterpart to G12. There can be a number of reasons for this, including differences in X-ray positions, deblending or non-deblending of photometry in crowded regions, and the fact that we match to a wider range of catalogs. For example, in several mismatched cases, we identify a single point-like counterpart in the HST Advanced Camera for Surveys (ACS) images, which in the ground-based images is merged with two or more very nearby objects into an apparent single source, identified as the counterpart by G12. A further check of the consistency between our associations those of G12 can be made by comparing the spectroscopic redshifts of the counterparts, when available. The G12 catalog contains 180 sources with reliable redshift from DEEP2. For 155 of these, we find the same redshift. For a further two (aegis_ 704 and aegis_762 in this paper) we assign the same counterpart but the redshift is different because we adopted a revised redshift value using DEEP3. Of the remaining 23 sources, there are 14 with spectroscopy in G12 that are not detected as significant X-ray sources in our reduction. For the final nine, we assign a different counterpart to G12, for the reasons discussed above, and hence have a different spectroscopic redshift.

\section{REDSHIFTS}

The AEGIS field has been the subject of a number of dedicated redshift surveys, most notably the DEEP2 and DEEP3 surveys (Davis et al. 2003; Cooper et al. 2011, 2012; Newman et al. 2013), with the Keck telescope. The outstanding multiwavelength photometry in the field also permits the determination of accurate photometric redshifts, and the spectroscopic data allow the calibration of those redshifts and estimates of their accuracy and reliability.

\subsection{Spectroscopic Redshifts}

A total of 353 sources in our AEGIS-XD X-ray sample have a reliable spectroscopic data, obtained from a variety of sources, which are listed in priority order in Table 9. The largest number (167) of these were derived from the DEEP2 redshift survey (Newman et al. 2013). DEEP2 was a magnitude-limited redshift survey (to $R_{\mathrm{AB}}=24.1$ ) covering approximately 2.8 square degrees over four fields. Among these fields, the AEGIS area features the most extensive multiwavelength coverage (Davis et al. 2007). Furthermore, the target selection in the AEGIS field was largely based on optical magnitude, unlike the other fields in DEEP2 where color selections were also applied to isolate high redshift galaxies. The redshift success for the AEGIS-XD sources targeted in DEEP2 is very high $(>77 \%)$. Note that we count only the spectra with redshift quality 3 and 4 as defined by Newman et al. (2013) as secure redshifts, ignoring lower qualities. On the other hand, the sampling rate of DEEP2 combined with selection against presumed stellar objects in the survey means that not all X-ray source counterparts brighter than the magnitude limit were covered. In addition, because the X-ray sources were not known at the time the DEEP2 survey was designed, they could not be targeted explicitly.

The DEEP3 survey (Cooper et al. 2011), an extension of DEEP2, addressed this issue by specifically targeting the counterparts of X-ray sources in the field regardless of their optical properties. DEEP3 provides an additional 89 spectroscopic redshifts for the AEGIS-XD survey. The success rate in DEEP3 $(\sim 51 \%)$ was lower than in DEEP2 because targets fainter than the DEEP2 magnitude limit were included. These spectra often failed to yield a secure redshift. A further important spectroscopic dataset was provided using the MMT/ Hectospec instrument (Coil et al. 2009), again explicitly targeting X-ray sources that were not already covered by DEEP2. This campaign provided a total of 81 secure redshifts for AEGIS-XD sources. Finally, a handful of spectroscopic redshifts of X-ray source counterparts have been obtained by other campaigns, for example the Canada-France Redshift Survey (Lilly et al. 1995), the Keck Lyman Break Galaxy (LBG) surveys of Steidel et al. (2003, 2004), and the SDSS (Ahn et al. 2012). Of these, SDSS provides six additional redshifts, and the LBG survey provides additional six. The five redshifts from CFRS were all duplicated in DEEP2/3 or the MMT surveys, so we use those in preference.

The grand total of 353 spectra implies a total spectroscopic completeness of the sample of $\sim 38 \%$. This is, however, a very strong function of optical magnitude, as can be seen from Figure 12. Only 20 spectroscopic redshifts have been obtained at magnitudes fainter than $R_{C}=24$, despite this being the peak of the magnitude distribution of the X-ray counterparts. This accounts for the relatively low spectroscopic completeness of the whole sample, despite major efforts in terms of spectroscopy in this field. A total of $111 \mathrm{X}$-ray source counterparts were targeted in the course of the various surveys without a reliable redshift being obtained.

\subsection{Photometric Redshifts}

Despite the intensive spectroscopy in this field, a relatively large fraction of the AEGIS-XD sources do not have spectroscopic redshifts. Photometric redshifts were therefore computed for the remaining sources with multiwavelength counterparts 
Table 8

Summary of Likelihood Ratio Matching Results for AEGIS-XD

\begin{tabular}{|c|c|c|c|c|c|c|c|c|c|c|c|}
\hline $\begin{array}{l}\text { Catalog } \\
\text { (1) }\end{array}$ & $\begin{array}{c}\text { Area/deg } \\
\text { (2) }\end{array}$ & $\begin{array}{l}N_{0} \\
(3)\end{array}$ & $\begin{array}{l}\sigma_{0} \\
(4)\end{array}$ & $\begin{array}{l}L_{\mathrm{th}} \\
(5)\end{array}$ & $\begin{array}{l}R \\
(6)\end{array}$ & $\begin{array}{c}C \\
(7)\end{array}$ & $\begin{array}{l}N_{\mathrm{X}} \\
(8)\end{array}$ & $\begin{array}{l}N_{\text {ID }} \\
(9)\end{array}$ & $\begin{array}{c}N_{\mathrm{NoID}} \\
(10)\end{array}$ & $\begin{array}{c}N_{\text {Multi }} \\
(11)\end{array}$ & $\begin{array}{l}N_{\text {Pri }} \\
(12)\end{array}$ \\
\hline RAINBOW $[3.6 \mu \mathrm{m}]$ & 0.26 & 65675 & 0.20 & 0.80 & 0.98 & 0.94 & 902 & 860 & 42 & 451 & 860 \\
\hline Subaru $R$ & 0.28 & 126709 & 0.20 & 1.18 & 0.95 & 0.80 & 936 & 788 & 148 & 435 & 37 \\
\hline Subaru $K$ & 0.08 & 10908 & 0.20 & 3.04 & 0.97 & 0.86 & 408 & 363 & 45 & 165 & 2 \\
\hline CFHTLS $i$ & 0.26 & 116686 & 0.30 & 1.10 & 0.95 & 0.77 & 828 & 670 & 158 & 401 & 1 \\
\hline MMT $i$ & 0.29 & 93412 & 0.30 & 1.03 & 0.94 & 0.75 & 937 & 752 & 185 & 370 & 1 \\
\hline
\end{tabular}

Note. Column 1: catalog name and detection band used to match counterparts. Column 2: area covered by both the multiwavelength data and deep X-ray data. Column 3: number of multiwavelength sources in the X-ray area. Column 4: $1 \sigma$ positional accuracy of multiwavelength catalog in arcseconds. Column 5: likelihood ratio threshold determined by the iterative procedure described in Section 4. Column 6: sample reliability, the mean of the individual reliabilities of each secure counterpart, as in Luo et al. (2010). Column 7: sample completeness, the sum of the reliabilities for all counterparts divided by the total number of X-ray sources. Column 8: total number of X-ray sources in the area covered by the multiwavelength data. Column 9: number of secure counterparts to X-ray sources. Column 10: number of X-ray sources without secure counterparts. Column 11: number of X-ray sources with more than one candidate counterpart within the 3".5 search radius. Column 12: number of X-ray sources assigned a primary counterpart in this band.

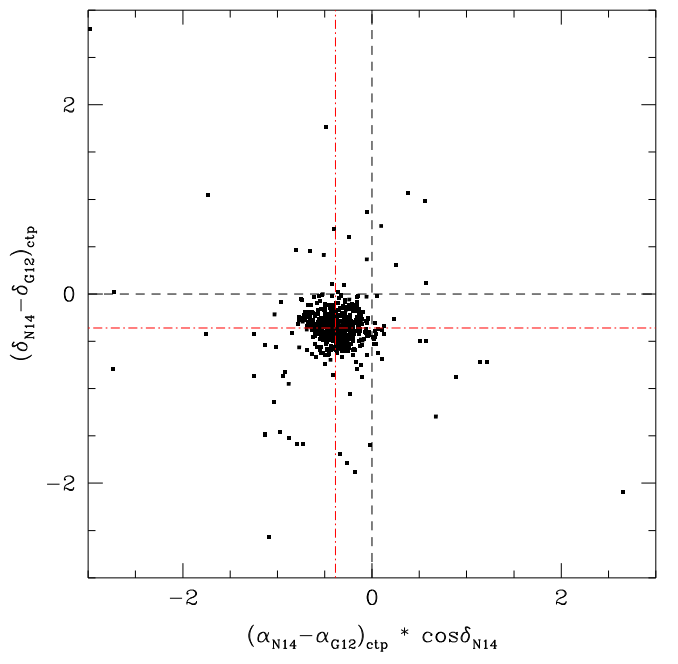

Figure 11. Positional offset between our counterparts and those of G12. A total of 595 sources are assigned both a counterpart in G12 and a secure counterpart in our work. The figure shows a systematic offset between the counterpart positions, which are presumably associated with the different astrometric frames used for the Rainbow database (our work) and DEEP2 (G12). After correction for this offset, the vast majority (572 of 595 or $97 \%$ ) have the same counterpart within a 0 ". 5 radius. A few show larger differences, indicating that our methodology finds a different counterpart, or has a significantly different position (e.g., due to the fact that we perform deblending).

using SED fitting. The photometric redshifts were computed following the analysis procedure described in Salvato et al. (2011), which takes into account knowledge of the optical morphology, optical variability, and X-ray emission to determine the most appropriate library and priors to be used. This method has been successfully applied in the COSMOS field (Salvato et al. 2009), the Lockman Hole (Fotopoulou et al. 2012), and the Extended Chandra Deep Field South (Hsu et al. 2014). More details regarding the adopted libraries can be found in Hsu et al. (2014).

Morphological classifications for the optical counterparts were taken from the Rainbow survey. There, the Hubble Space Telescope-ACS images were primarily used for the separation of the sources into point-like and extended. When the ACS data were not available, the analysis was performed on the Subaru $R_{c}$ band image (FWHM $\left.\sim 0^{\prime \prime} .7\right)$. The motivation for this is that
Table 9

Spectroscopic Redshifts

\begin{tabular}{lcccc}
\hline \hline Survey $^{\mathrm{a}}$ & $N_{\text {targ }} \mathrm{b}$ & $N_{\text {spec }} \mathrm{c}$ & $N_{\text {used }} \mathrm{d}$ & $N_{\text {star }} \mathrm{e}$ \\
\hline DEEP3 & 174 & 89 & 89 & 3 \\
DEEP2 & 223 & 172 & 167 & 0 \\
MMT & 162 & 93 & 91 & 10 \\
CFRS & 5 & 5 & 0 & 0 \\
SDSS & 14 & 14 & 6 & 1 \\
LBG & 7 & 7 & 6 & 0 \\
\hline Total & $\ldots$ & 464 & 339 & 14 \\
\hline
\end{tabular}

Notes.

${ }^{\text {a }}$ The redshift origins are listed in priority order (i.e., the redshift is taken preferentially from DEEP3 if available, then DEEP2 and so on down to SDSS, which has the lowest priority). Secure redshifts and stellar identifications are those with redshift quality 3 or 4 in the catalogs. Lower quality flags are assumed to be redshift failures.

${ }^{\mathrm{b}}$ Number of AEGIS-XD counterparts targeted for spectroscopy.

${ }^{c}$ Secure redshifts and stellar identifications are those with redshift quality 3 or 4 in the catalogs. Lower quality flags are assumed to be redshift failures.

${ }^{\mathrm{d}}$ Unique reliable spectroscopic identifications used in this work.

${ }^{\text {e }}$ Number of spectroscopically confirmed stars. The difference between $N_{\text {used }}$ and $N_{\text {star }}$ provides the number of galaxies.

optically extended sources (i.e., resolved galaxies) are likely to be better modeled with a galaxy dominated template. Optical point sources and/or variable sources are candidate type 1 QSOs, which are better fit with an AGN template. We classified 536 sources as optically extended (EXTNV) and the remaining 401 as point-like or unresolved (QSO variable or QSOV). ${ }^{20}$ The EXTNV group was also split on the basis of the soft X-ray flux, with 530 sources fainter than $\mathrm{F}_{(0.5-2 \mathrm{keV})}=8 \times 10^{-15}$ $\mathrm{erg} \mathrm{cm} \mathrm{cm}^{-2} \mathrm{~s}^{-1}$ and the remaining six brighter than that.

Source variability can be a major issue in determining the photometric redshifts for type I AGN because the multiband data were usually taken at different epochs (i.e., there can be significant flux-offsets worsening the SED fits or causing the wrong template to be selected). In addition, in many cases the photometry in a given band was produced by adding the results

\footnotetext{
$\overline{{ }^{20}}$ We adopt the same classification as defined in Salvato et al. (2009) for ease of comparison.
} 


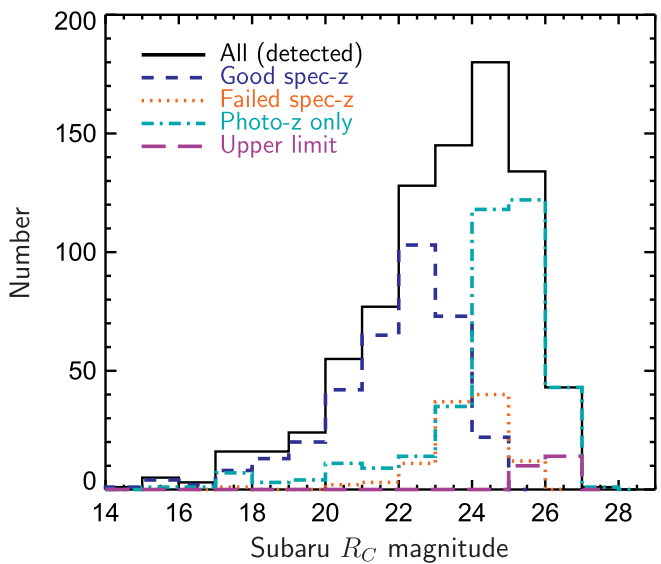

Figure 12. Subaru $R_{C}$ magnitude distribution of the AEGIS-XD counterparts. The distribution peaks at $R_{C} \sim 24$. The vast majority of successful spectroscopic redshifts are at brighter magnitudes than this. Redshift failures start to rise sharply at $R_{C}>23$, below which we must largely rely on photometric redshifts. The upper limits curve refers to the upper limit on the magnitude in the case where there is no detected counterpart in the Subaru imaging.

from different observation runs separated in time, which means that a multi-epoch variability analysis was not possible. However, we do find that 59 sources have a clear offset (typically 0.5 mag and up to $\sim 1$ ) when comparing photometry from the same or similar filters, suggesting variability. Visual inspection shows that 38 of these sources are point-like, and while the morphological classification is a strong function of magnitude and image resolution, this suggests that the variability is likely real and due to the QSO or stellar nature of the source. For the remainder, which are classified as optically extended, half are located close to nearby stars and the variability is likely more related to variation in the flux of the stars or the background in their vicinity. For lack of further information, all 59 apparently variable sources have been flagged, as the variability might have a significant effect on the redshift determination. As in Salvato et al. (2011), we used the LePhare code (Ilbert et al. 2006) to compute the photometric redshifts. The first step was to correct the photometry for Galactic extinction using a median $E(B-V)=0.04$ (see second-to-last column of Table 7, as in Barro et al. 2011a). Then we searched for possible zeropoint offsets that could affect the accuracy of the photometric redshift. To do this we computed the photometric redshift of a sample of normal galaxies (i.e., non X-ray detected) with the reliable spectroscopic redshift available. For each source, we kept the redshift fixed and searched for the best fitting template in the library of normal galaxies used in Ilbert et al. (2009). Then, for each photometric band, we computed the average difference between the photometry of all the sources and the photometry of the template. In this way, we can correct for second-order problems in the photometric calibration. We adopted these zeropoints (reported in the last column of Table 7) when computing the photometric redshifts for the X-ray selected sources. We note that the zeropoint corrections depend on the templates used, and different libraries could provide slightly different values for the correction. For this reason we do not apply these corrections to the photometric catalog released with this paper. Furthermore, the same procedure could not be applied directly to the X-ray sources because (a) variability could affect the results and (b) the relative host/AGN contribution to the $\mathrm{SED}$ in a given band is unknown. Ignoring these facts can

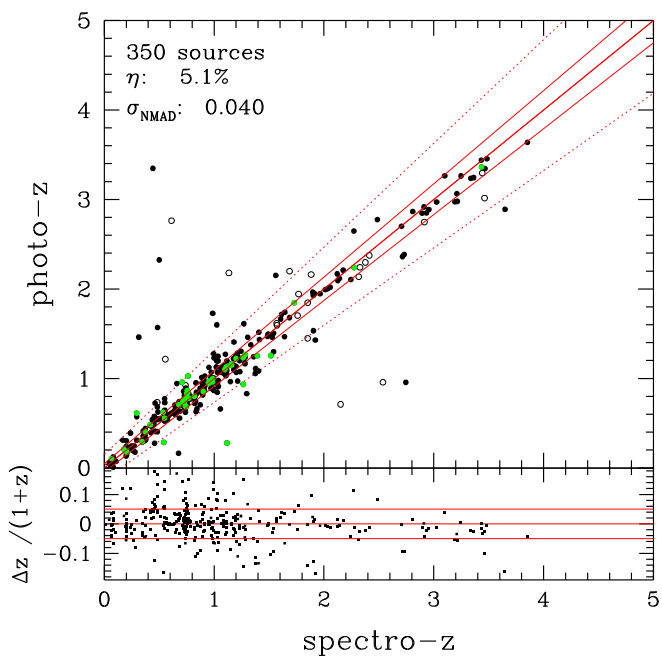

Figure 13. Comparison between spectroscopic and photometric redshifts for the extragalactic spectroscopic sample. Filled dots indicate sources with a possible unique redshift solution, while open circles represent sources for which there is at least a second significant peak in the redshift probability distribution. In green we show the sources within the CANDELS area, where deeper and better resolved NIR data are available, yielding superior photo- $z$ results. The solid lines correspond to $z_{\text {phot }}=z_{\text {spec }}$ and $z_{\text {phot }}= \pm 0.05\left(1+z_{\text {spec }}\right)$, respectively. The dotted lines limit the locus where $z_{\text {phot }}= \pm 0.15\left(1+z_{\text {spec }}\right)$.

potentially introduce a greater uncertainty in the zeropoints when applying a relatively limited number of templates.

We compute the photometric redshift for the EXTNV sources using the new hybrid templates of Hsu et al. (2014). These templates are tuned for sources dominated by galaxies, with special care given in reproducing the emission lines. For the QSOV sample we used the AGN-dominated hybrids of Salvato et al. (2009). Different absolute magnitude priors were considered for EXTNV $\left(-24<M_{B}<-8\right)$ and QSOV $\left(-30<M_{B}<-20\right)$ sources, respectively. In order to search for stars in the sample, a stellar library was also used to fit the data. Whenever a source in the QSOV sample was better fit with the stellar template it was classified as a star. There are 21 sources that satisfy this criterion $(\sim 3.1 \%$ of the entire sample), with 12 of these also spectroscopically confirmed. Turning to the extragalactic sources, the overall accuracy for the spectroscopic sample, measured by the normalized mean absolute deviation, is $\sigma_{\mathrm{NMAD}}=0.040$, with an outlier fraction of $\eta=5.1 \%$ (Figure 13$)$. The accuracy is slightly better $\left(\sigma_{\mathrm{NMAD}}\right.$ $=0.030$, with an outlier fraction of $\eta=3.8 \%$, where deeper and better resolved NIR photometry from CANDELS is available). A close look at the outliers revealed that most of those for which the photometric redshift solution was not unique (empty circles in the figure) had the correct solution in the second higher peak. The use of a redshift probability distribution function (made publicly available with this work) is recommended. The remaining outliers can be explained by blending with nearby sources for which the low resolution of the ground-based imaged misplace the sources in the "EXTNV" group, rather than in the "QSOV," thus adopting the wrong templates or priors. The effect of the resolution of the images used for the classification has been addressed in Hsu et al. (2014). These authors demonstrate that the fraction of outliers increases significantly when using the morphological classification from the ground-based images rather than from the space-based ones. We break down the results in redshift, magnitude, and type in Table 10. The outlier fraction and 
Table 10

Accuracy of Photometric Redshifts

\begin{tabular}{|c|c|c|c|c|c|c|}
\hline \multirow[t]{2}{*}{ Sub Sample } & \multicolumn{3}{|c|}{ QSOV } & \multicolumn{3}{|c|}{ EXTNV } \\
\hline & $\begin{array}{l}\text { N. of } \\
\text { Sources } \\
\quad(1)\end{array}$ & $\begin{array}{c}\eta(\%) \\
(2)\end{array}$ & $\begin{array}{c}\sigma_{\mathrm{NMAD}} \\
(3)\end{array}$ & $\begin{array}{c}\text { N. of } \\
\text { Sources } \\
\quad(1)\end{array}$ & $\begin{array}{c}\eta(\%) \\
(2)\end{array}$ & $\begin{array}{c}\sigma_{\mathrm{NMAD}} \\
(3)\end{array}$ \\
\hline$z<1.2$ & 53 & 11.3 & 0.067 & 201 & 3.5 & 0.034 \\
\hline$z>1.2$ & 72 & 5.6 & 0.059 & 24 & 4.2 & 0.027 \\
\hline$R<22 \mathrm{mag}$ & 41 & 7.3 & 0.067 & 111 & 2.7 & 0.040 \\
\hline$R>22 \mathrm{mag}$ & 84 & 8.3 & 0.061 & 114 & 4.4 & 0.028 \\
\hline Combined & 125 & 8.0 & 0.063 & 225 & 3.6 & 0.033 \\
\hline
\end{tabular}

Note. (1) Number of sources, (2) outlier fraction, and (3) normalized mean absolute deviation. QSOV refers to optically point-like and/or variable sources. EXTNV refers to optically extended and non-variable sources.

uncertainties in the QSOV sample are larger, partly due to the degeneracies associated with the typical power-law SED of this subsample. This is a general problem for photometric redshifts for AGN, which can be mitigated when narrower filters sensitive to strong emission lines are used in the photo- $z$ determination (e.g., Salvato et al. 2009; Cardamone et al. 2010; Hsu et al. 2014).

The full probability distribution function for the photometric redshifts is made available for all sources in our catalog (see Appendix). We caution that the errors associated with the photometry can easily be underestimated, with the result that the $1-3 \sigma$ errors associated with the photometric redshift can also be underestimated. This is true for photometric redshifts in general (see Dahlen et al. 2013 for a recent test with a sample of normal galaxies in the CANDELS fields). We verified that the situation is similar for our sample where $z_{\text {phot }}-1 \sigma<z_{\text {spec }}<z_{\text {phot }}+1 \sigma$ only for $57 \%$ of the sources, and only $79 \%$ of the sample the spectroscopic sample is within the $3 \sigma$ error of the photometric redshift. Thus, while using the $3 \sigma$ errors associated with photometric redshifts provides a reasonably accurate estimate of the uncertainty for most of the sources, we recommend using the entire redshift probability distribution function for a more complete analysis.

\subsection{Redshift Distribution}

The redshift distribution of our X-ray sources (after excluding the stars) is shown in Figure 14, distinguishing between spectroscopic and photometric redshifts. There is a peak in the spectroscopic redshift distribution around $z \sim 0.7$, which is also seen in the photometric redshift distribution, presumably due to a large-scale structure at this redshift in the field. The redshift distribution shows clearly that the spectroscopic completeness is a very strong function of redshift, with the sample being highly spectroscopically complete at $z<1$ (210 of 303), and highly incomplete above this redshift (140 of 613). This shows the great importance of computing accurate photo- $z$ when considering AGN evolution and/or host galaxy properties at high redshift.

Figure 14 also shows in blue the sources that are characterized by at least one further peak in the redshift solution (200 sources, $23 \%$ of the sample). Sources shown in red are those for which a single peak in the redshift solution is found, but for which the peak $P(z)$ is lower than $50 \%$ (34 sources, $6 \%$ ), with a distribution over a broad redshift range.

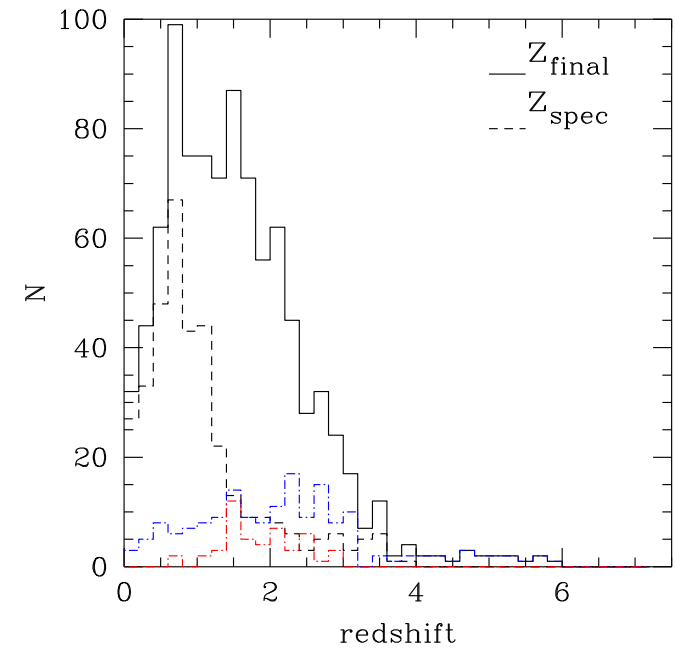

Figure 14. Redshift distribution for the X-ray sources. The black dashed line indicates the distribution for the sources with secure spectroscopic redshift; in black solid line the same distribution is added to the rest of the sources for which only a photo- $z$ is available. In red we indicate the sources for which there are at least two peaks in the redshift solution, while in blue we show the distribution of the sources for which there is a single peak, but with a low value of $P(z)$.

The majority of the high redshift sources (e.g., at $z>3.5$ ) are among these poorly defined sources. We find $14 \mathrm{X}$-ray sources at $z=3.5-4.5$ in the AEGIS field (two spectroscopically confirmed), and 15 at redshift $z>4.5$, although all of the latter show a secondary peak at a lower redshift. In this regime the photometric redshifts are strongly dependent on the upper limits adopted, making the results unstable. The high redshift nature of these sources thus needs to be assessed carefully, ideally via spectroscopy or possibly with deeper photometry (e.g., Venemans et al. 2007). For the time being the photometric redshifts should be considered with the associated errors and preferably with the full $P(z)$.

Figure 15 shows our sample in the $L_{\mathrm{X}}-z$ plane. The left panel distinguishes between sources with spectroscopic redshifts and those with photometric redshifts. This clearly shows that that vast majority of sources at $z>1.5$ do not have spectroscopic redshift, making the photo- $z$ essential. Obtaining accurate photo- $z$, as in our work, is thus crucial for the investigation of AGN properties at high redshift. The AEGIS survey alone provides reasonably good sampling of the population in the luminosity range $10^{42-44}$ up to around $z-3$, after which it becomes incomplete at the faint end. This can be improved by combining it with deeper surveys such as the CDF-N and, particularly, the CDF-S. Similarly, the luminosity range above $10^{44} \mathrm{erg} \mathrm{s}^{-1}$, above $L_{*}$, the knee in the XLF, is relatively sparsely sampled by our data, and larger area surveys are required to determine the bright end of the XLF with good accuracy. The right panel of Figure 15 shows the objects color coded by the best-fit template fitted to the multiwavelength photometry during the photo- $z$ determination. There is a considerable mix of template types depending on whether the light at longer wavelengths is dominated by the AGN, the galaxy, or a mixture. Previous works using the same photometric redshift methods used here have shown good agreement between the SED type and the spectroscopic classification, where available (Salvato et al. 2009; Lusso et al. 2010, 2012; Civano et al. 2012) 

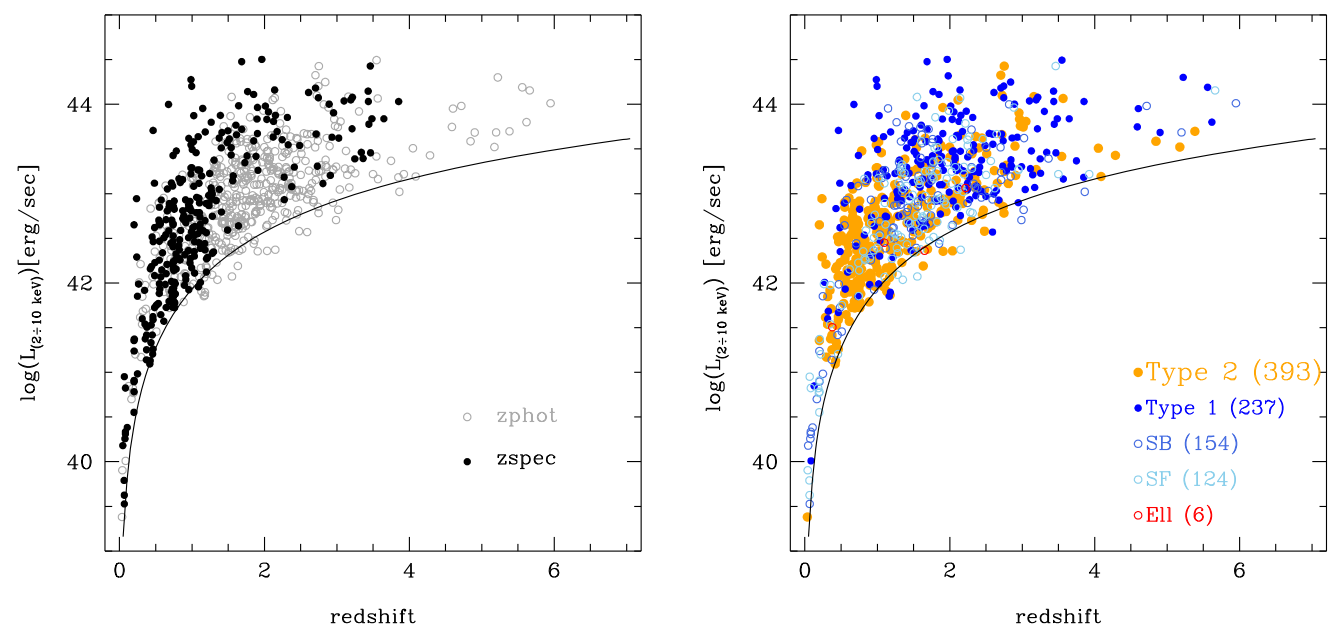

Figure 15. Left panel: $\mathrm{X}$-ray luminosity in the rest frame $2-10 \mathrm{keV}$ band as a function of spectroscopic (black filled circles) or photometric redshift (gray open circles). Right panel: same plot but this time the sources are color coded on the basis of the best-fit template to the photometric data. Here filled symbols indicate sources best-fit by an AGN template (type 1 or 2), or a hybrid with some contribution (from 10\% to 100\%) from an AGN. Galaxy templates are shown as open symbols and include Ellipticals (Ell), various spiral/irregular templates (SF), and starbursts (SB). The number of sources of each SED type are also reported in the figure. Note that the sources fit by an AGN/hybrid are on average more luminous in the X-ray, as might be expected. Even if the optical/IR SED is better fit with a galaxy template, however, the X-rays will in the vast majority of cases be dominated by an AGN. In both figures, the black solid curve corresponds to a flux limit of $f_{X}(2-10 \mathrm{keV})=2.5 \times 10^{-16} \mathrm{erg} \mathrm{s}^{-1} \mathrm{~cm}^{-2}$, where the $2-10 \mathrm{keV}$ sensitivity curve drops to $1 \%$ of the maximum value. For the calculation of $2-10 \mathrm{keV}$ luminosity we used the $0.5-10 \mathrm{keV}$ flux, k-corrected using a power-law X-ray spectrum with spectral index $\Gamma=1.4$. Because the $0.5-10 \mathrm{keV}$ band is more sensitive than the 2-10 keV band, a few sources appear below the line corresponding to the flux limit.

\section{SUMMARY}

A catalog of X-ray sources detected in the deep (800 ks) Chandra imaging of the AEGIS field has been presented. This is currently the third deepest X-ray survey in existence, after the Chandra Deep Fields North and South. A total of 937 X-ray sources have been detected down to a Poisson false probability of $4 \times 10^{-6}$, calculated based on the counts detected in a PSF-sized detection cell and a local background, following the methodology of L09. The source detection algorithm enables an accurate determination of the sensitivity of the observations over the field using the technique of Georgakakis et al. (2008b), and hence the catalog can be used effectively when statistical investigations requiring corrections for completeness are needed (e.g., luminosity functions). We have identified multiwavelength counterparts to our sources using a wide variety of complementary data in this field, ranging from the UV to the mid-infrared. Using a likelihoodbased association method, we find possible counterparts for 929 of 937 (or $99 \%$ ) of the X-ray sources, the vast majority from the deep Spitzer/IRAC imaging in the field. We note, however, that the statistical reliability of likelihood-based associations is not $100 \%$, so the notional completeness of the counterpart identifications is closer to $94 \%$ in a statistical sense. Of the X-ray source counterparts, $353(\sim 38 \%)$ have a reliable spectroscopic redshift, mostly from Keck spectroscopy in the DEEP2 and DEEP3 surveys, supplemented by a significant number from MMT/Hectospec spectroscopy. For all X-ray source associations, we performed multiwavelength photometry in up to 35 bands using the methodology pioneered in the Rainbow database (Barro et al. 2011a). This provides SEDs for the sources and furthermore enables accurate photometric redshifts to be determined using the methodology of Salvato et al. (2011). This is tuned particularly for X-ray sources detected in deep surveys, which mostly comprise AGN. Despite greater difficulties and uncertainties associated with determining photo- $z$ for such sources, the reliability and accuracy of the photometric redshifts is excellent, with an outlier fraction of just $\eta=5 \%$ and $\sigma=0.04$. Even better results $\eta=4 \%$ and $\sigma=0.03$ are reached in the CANDELS area where deeper and superior NIR data are available. The AEGIS-XD dataset lies in a unique area of parameter space in terms of deep X-ray surveys and the excellence of the redshift determinations, and the supporting multiwavelength data make it a powerful tool to investigate the AGN population. The dataset described here has already been used to investigate the colors of AGN hosts (Georgakakis 2014a), AGN clustering (Georgakakis 2014b), Compton-thick AGN (Brightman et al. 2014), and the evolution of AGN obscuration (Buchner et al. 2015), typically in combination with other deeper and/or wider datasets. Further work investigating these and related phenomena should greatly enhance our knowledge of black hole growth over cosmic time, and its relationship to galaxy evolution.

All of our catalogs, including the detailed X-ray information, multiwavelength identifications, aperture-matched photometry, and redshift information (including the SED fits and photometric redshift $P(z))$ are released publicly, as described in the Appendix to this paper.

We thank those who built and operate the Chandra X-ray observatory so successfully. We acknowledge financial support from Chandra grant G08-9129 A, NSF grant AST-0808133, US National Science Foundation via grant AST-0806732. P. G. P.-P. acknowledges support from the Spanish Programa Nacional de Astronomía y Astrofísica under grant AYA201237727. This work made use of the Rainbow Cosmological Surveys Database, which is operated by the Universidad Complutense de Madrid (UCM) partnered with the University of California Observatories at Santa Cruz (UCO/Lick,UCSC).

Facilities: CXO(ACIS), CFHT, GALEX, Hale, HST, Keck: I, Keck: II, Mayall, MMT, Sloan, Subaru, Spitzer(IRAC). 


\begin{tabular}{|c|c|c|c|c|c|c|c|c|c|c|c|c|c|c|}
\hline \multirow[b]{2}{*}{$\begin{array}{l}\text { XID } \\
(1)\end{array}$} & \multirow[b]{2}{*}{$\begin{array}{c}\text { IAU Name } \\
\text { (2) }\end{array}$} & \multirow{2}{*}{$\begin{array}{c}\text { R.A. } \\
\text { (J2000) } \\
(3)\end{array}$} & \multirow{2}{*}{$\begin{array}{c}\text { Decl. } \\
(\text { J2000) } \\
(4)\end{array}$} & \multirow[b]{2}{*}{$\begin{array}{l}\text { Pos. err } \\
(5)\end{array}$} & \multirow[b]{2}{*}{$\begin{array}{l}\text { OAA } \\
(6)\end{array}$} & \multicolumn{2}{|c|}{ FB cts } & \multicolumn{2}{|c|}{ SB cts } & \multicolumn{2}{|c|}{$\mathrm{HB}$ cts } & \multicolumn{2}{|c|}{ UB cts } & \multirow{2}{*}{$\begin{array}{c}\text { Detection } \\
\text { Bands } \\
(15)\end{array}$} \\
\hline & & & & & & $\begin{array}{l}N \\
(7)\end{array}$ & $\begin{array}{c}\text { B } \\
(8)\end{array}$ & $\begin{array}{l}\mathrm{N} \\
(9)\end{array}$ & $\begin{array}{c}\text { B } \\
(10)\end{array}$ & $\begin{array}{c}\mathrm{N} \\
(11)\end{array}$ & $\begin{array}{c}\text { B } \\
(12)\end{array}$ & $\begin{array}{c}\mathrm{N} \\
(13)\end{array}$ & $\begin{array}{c}\text { B } \\
(14)\end{array}$ & \\
\hline aegis_001 & AEGISXD J141805.22+522510.6 & 214.521757 & 52.419615 & 0.87 & 12.11 & 125 & 72.99 & 57 & 21.72 & 68 & 53.27 & 43 & 33.92 & FSH \\
\hline aegis_002 & AEGISXD J141807.03+522523.0 & 214.529298 & 52.423079 & 0.57 & 11.90 & 245 & 76.40 & 105 & 21.80 & 135 & 54.80 & 61 & 34.16 & FSHU \\
\hline aegis_003 & AEGISXD J141816.27+522524.7 & 214.567822 & 52.423529 & 0.87 & 11.97 & 128 & 43.68 & 58 & 12.46 & 71 & 32.46 & 36 & 20.80 & FSH \\
\hline aegis_004 & AEGISXD J141826.59+522602.0 & 214.610827 & 52.433892 & 0.87 & 11.68 & 142 & 86.52 & 41 & 24.20 & 105 & 64.69 & 55 & 40.69 & $\mathrm{FH}$ \\
\hline aegis_005 & AEGISXD J141813.90+522625.0 & 214.557935 & 52.440278 & 0.87 & 10.93 & 175 & 123.78 & 54 & 32.87 & 124 & 94.25 & 72 & 60.56 & FS \\
\hline aegis_006 & AEGISXD J141757.02+522631.3 & 214.487603 & 52.442038 & 0.57 & 10.85 & 183 & 52.06 & 66 & 14.05 & 120 & 40.54 & 49 & 26.50 & FSHU \\
\hline aegis_007 & AEGISXD J141821.24+522655.7 & 214.588520 & 52.448820 & 0.87 & 10.61 & 232 & 157.02 & 77 & 42.63 & 160 & 119.96 & 94 & 77.03 & FS \\
\hline aegis_008 & AEGISXD J141822.87+522709.5 & 214.595307 & 52.452642 & 0.87 & 10.44 & 240 & 154.97 & 128 & 42.36 & 106 & 117.96 & 72 & 76.39 & FS \\
\hline aegis_009 & AEGISXD J141829.77+522709.6 & 214.624081 & 52.452675 & 0.87 & 10.75 & 163 & 100.76 & 44 & 27.15 & 127 & 77.76 & 71 & 49.70 & FH \\
\hline aegis_010 & AEGISXD J141804.84+522740.2 & 214.520174 & 52.461183 & 0.57 & 9.61 & 213 & 105.52 & 93 & 27.81 & 118 & 81.69 & 66 & 54.83 & FS \\
\hline
\end{tabular}

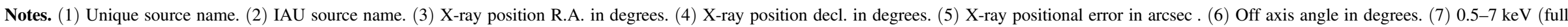

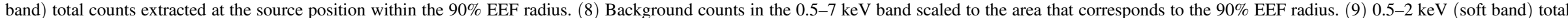

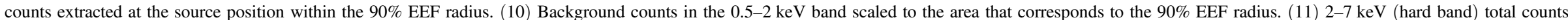

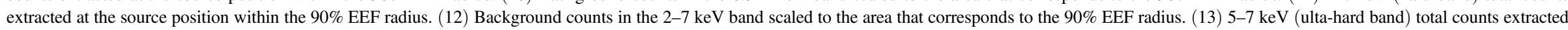

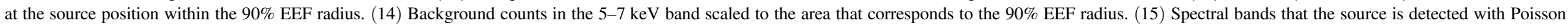
background fluctuation probability $<4 \times 10^{-6}$. The letters correspond to full band $(\mathrm{F})$, soft band $(\mathrm{S})$, hard band $(\mathrm{H})$, and ultrahard band $(\mathrm{U})$.

(This table is available in its entirety in machine-readable form.) 
Table 12

Chandra AEGIS-X Source Catalog: Source Fluxes and HRs

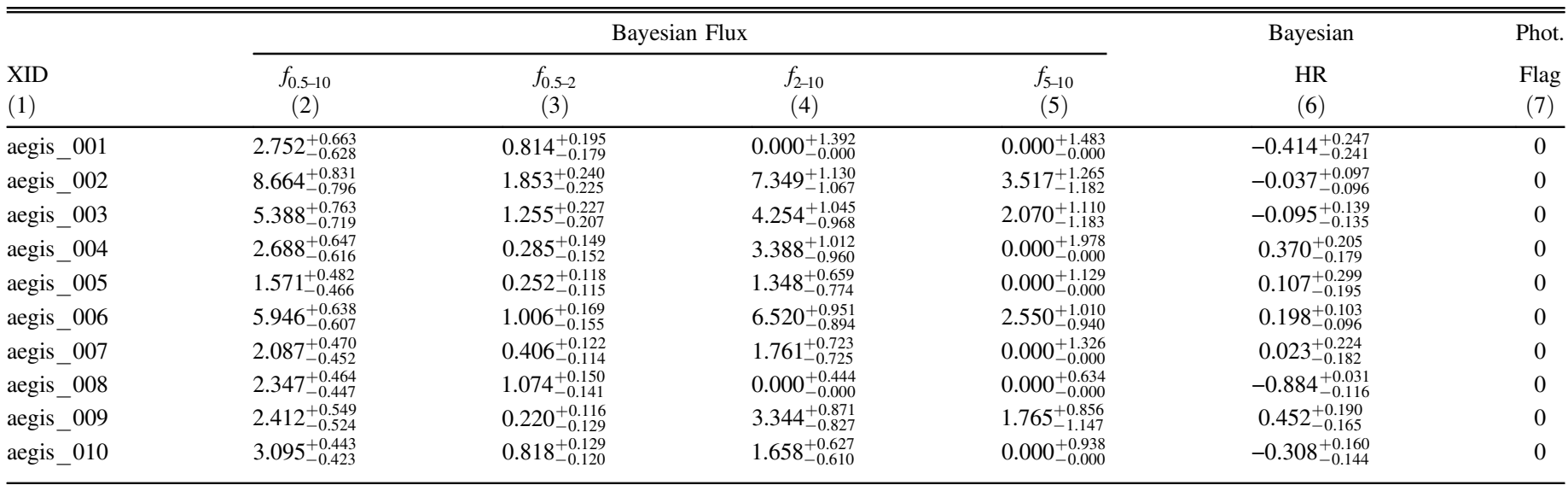

Notes. (1)Unique source name. (2) Flux in the $0.5-10 \mathrm{keV}$ band in units of $10^{-15} \mathrm{erg} \mathrm{s}^{-1} \mathrm{~cm}^{-2}$ estimated using the Bayesian methodology of L09. (3) Flux in the 0.5-2 keV band in units of $10^{-15} \mathrm{erg} \mathrm{s}^{-1} \mathrm{~cm}^{-2}$ estimated using the Bayesian methodology of L09. (4) Flux in the 2-10 keV band in units of $10^{-15} \mathrm{erg} \mathrm{s}^{-1} \mathrm{~cm}^{-2}$ estimated using the Bayesian methodology of L09. (5) Flux in the 5-10 keV band in units of $10^{-15} \mathrm{erg} \mathrm{s}^{-1} \mathrm{~cm}^{-2}$ estimated using the Bayesian methodology of L09. (6) Hardness ratio determined by BEHR (Park et al. 2006) using the counts in the $0.5-2$ and $2-7 \mathrm{keV}$ spectral bands. (7) Quality of the X-ray photometry. A flag of "1" indicates the presence of a nearby source that may be contaminating the photometry. A flag of "2" indicates that another source was detected with in the 90\% EEF and that the photometry is likely heavily contaminated and the source position uncertain. All other sources have a flag of " 0. "

(This table is available in its entirety in machine-readable form.)

\section{APPENDIX \\ CATALOGS AND DATA RELEASE}

The catalogs described in this paper are available via the journal and the public websites at MPE www.mpe.mpg.de/ XraySurveys and via Rainbow (http://rainbowx.fis.ucm.es/ Rainbow_Database/Home.html). Specifically, we release the following products:

1. The X-ray source catalogs

2. The optical/NIR/MIR association catalog for the X-ray sources with corresponding multiband photometry
3. The redshifts, including spectroscopic redshifts, photometric redshifts, and redshift probability distributions.

In this Appendix, we show extracts from each of the catalogs, with the information provided in the electronic edition of the journal. The full information is provided on the website in FITS format.

\section{A.1. X-ray Catalogs}

A subset of the basic X-ray properties of the AEGIS-XD sources are given in Tables 11 and in 12. In the first we provide

Table 13

Optical and IR Counterparts to the AEGIS-X Sources

\begin{tabular}{|c|c|c|c|c|c|c|c|c|}
\hline $\mathrm{XID}^{\mathrm{a}}$ & Index No. ${ }^{b}$ & $\begin{array}{l}\text { Rainbow }^{\mathrm{c}} \\
\text { (Rainbow) }\end{array}$ & $\begin{array}{l}\text { X-ray R.A. }{ }^{\mathrm{d}} \\
(\mathrm{J} 2000)\end{array}$ & $\begin{array}{c}\text { X-ray Decl. }{ }^{\mathrm{d}} \\
(\mathrm{J} 2000)\end{array}$ & $\begin{array}{l}\text { Ctrp. R.A. } \\
\text { (J2000) }\end{array}$ & $\begin{array}{c}\text { Ctrp. Decl. }^{\mathrm{e}} \\
\quad(\mathrm{J} 2000)\end{array}$ & Prim_Match ${ }^{\mathrm{f}}$ & Robust_Ctrp. ${ }^{\mathrm{g}}$ \\
\hline aegis_001 & 1 & aegis_001 & 214.5217932 & 52.4196191 & 214.5223028 & 52.4197497 & RAINBOW & 1 \\
\hline aegis_002 & 4 & aegis_002_3 & 214.5293342 & 52.4230831 & 214.5295632 & 52.4231009 & RAINBOW & 1 \\
\hline aegis_003 & 5 & aegis_003 & 214.5678582 & 52.4235331 & 214.5680005 & 52.4232972 & RAINBOW & 1 \\
\hline aegis_004 & 6 & aegis_004_1 & 214.6108522 & 52.4338898 & 214.6103281 & 52.4330603 & none & 0 \\
\hline aegis_005 & 10 & aegis_005_3 & 214.5579712 & 52.4402821 & 214.5581131 & 52.4405130 & RAINBOW & 1 \\
\hline aegis_006 & 11 & aegis_006_1 & 214.4876392 & 52.4420421 & 214.4879252 & 52.4420215 & RAINBOW & 1 \\
\hline aegis_007 & 14 & aegis_007_2 & 214.5885562 & 52.4488241 & 214.5884986 & 52.4490495 & RAINBOW & 1 \\
\hline aegis_008 & 15 & aegis_008 & 214.5953432 & 52.45264601 & 214.5951844 & 52.4524551 & RAINBOW & 1 \\
\hline aegis_009 & 16 & aegis_009_1 & 214.6241172 & 52.4526791 & 214.6241732 & 52.4526771 & RAINBOW & 1 \\
\hline aegis_010 & 18 & aegis_010 & 214.5202102 & 52.4611871 & 214.5203175 & 52.4612681 & RAINBOW & 1 \\
\hline
\end{tabular}

Notes.

${ }^{\mathrm{a}} \mathrm{X}$-ray Identification.

${ }^{\mathrm{b}}$ Index number used to identify files in online database.

${ }^{\mathrm{c}}$ Object Identification in Rainbow.

${ }^{\mathrm{d}} \mathrm{X}$-ray position, after registration to the Rainbow astrometry.

e Counterpart position.

f Band first used to identify multiwavelength counterpart; "none" indicates no counterpart identified.

${ }^{\mathrm{g}}$ Flag indicating whether the counterpart association is considered secure (1) or not (0) according to the likelihood thresholds listed in Table 8.

(This table is available in its entirety in machine-readable form.) 
Table 14

Extract from the AEGIS-XD Photometric Catalog

\begin{tabular}{|c|c|c|c|c|c|c|c|c|c|}
\hline $\begin{array}{l}\text { Rainbow } \\
\text { (1) }\end{array}$ & $\begin{array}{l}\text { Index No. } \\
\text { (2) }\end{array}$ & $\begin{array}{l}\text { Ctrp. R.A. } \\
\text { (3) }\end{array}$ & $\begin{array}{c}\text { Ctrp. Decl. } \\
\text { (4) }\end{array}$ & $\begin{array}{l}\mathrm{R} \\
(5)\end{array}$ & $\begin{array}{l}\operatorname{err}_{R} \\
(6)\end{array}$ & $\begin{array}{l}\ldots \\
(\ldots)\end{array}$ & $\begin{array}{l}\ldots \\
(\ldots)\end{array}$ & $\begin{array}{l}\text { FUV } \\
(73)\end{array}$ & $\begin{array}{c}\operatorname{err}_{\mathrm{FUV}} \\
(74)\end{array}$ \\
\hline aegis_001 & 1 & 214.5223028 & 52.4197497 & 24.45 & 0.05 & $\ldots$ & $\ldots$ & -99.00 & -99.00 \\
\hline aegis_002_3 & 4 & 214.5295632100 & 52.4231009 & 24.64 & 0.05 & $\ldots$ & $\ldots$ & -99.00 & -99.00 \\
\hline aegis_004_1 & 6 & 214.6103281 & 52.4330603 & 24.65 & 0.05 & $\ldots$ & $\ldots$ & -99.00 & -99.00 \\
\hline aegis_005_3 & 10 & 214.55811309 & 52.44051303 & 25.27 & 0.08 & $\ldots$ & $\ldots$ & -99.00 & -99.00 \\
\hline aegis_006_1 & 11 & 214.4879252 & 52.4420215 & 23.95 & 0.07 & $\ldots$ & $\ldots$ & -99.00 & -99.00 \\
\hline aegis_009_1 & 16 & 214.6241732 & 52.4526771 & 22.60 & 0.03 & $\ldots$ & $\ldots$ & -99.00 & -99.00 \\
\hline aegis_010 & 18 & 214.5203175 & 52.4612681 & 23.87 & 0.04 & $\ldots$ & $\ldots$ & -99.00 & -99.00 \\
\hline
\end{tabular}

Note. Excerpt from the photometric catalog for AEGIS-XID; it is published in its entirety in the electronic edition of the journal. Column 2: index number used to identify files in online database; Column 1: optical identifier number from the Rainbow catalog; Columns 3 and 4: R.A. and decl. in degrees of the counterpart; Column 5 and following odd columns: AB magnitude in the filters listed in Table 7; Column 6 and following even columns: associated photometric errors.

(This table is available in its entirety in machine-readable form.)

Table 15

Extract from the AEGIS-XD Redshift Catalog

\begin{tabular}{lccccccccccccccc}
\hline \hline $\begin{array}{l}\text { Rainbow } \\
(1)\end{array}$ & $\begin{array}{c}\text { Index No. } \\
(2)\end{array}$ & $\begin{array}{c}z_{s} \\
(3)\end{array}$ & $\begin{array}{c}z_{\text {conf }}(4) \\
\text { (4) }\end{array}$ & $\begin{array}{c}z_{\text {ref. }} \\
(5)\end{array}$ & $\begin{array}{c}\mathrm{Nb} \\
(6)\end{array}$ & $\begin{array}{c}z_{p 1} \\
(7)\end{array}$ & $\begin{array}{c}z_{p 1} 168 \\
(8)\end{array}$ & $\begin{array}{c}z_{p 1} \mathrm{U} 68 \\
(9)\end{array}$ & $\begin{array}{c}z_{p 1} 190 \\
(10)\end{array}$ & $\begin{array}{c}z_{p 1} \mathrm{U} 90 \\
(11)\end{array}$ & $\begin{array}{c}P\left(z_{p 1}\right) \\
(12)\end{array}$ & $\begin{array}{c}\operatorname{Mod}_{1} \\
(13)\end{array}$ & $\begin{array}{c}z_{p 2} \\
(14)\end{array}$ & $\begin{array}{c}P\left(z_{p 2}\right) \\
(15)\end{array}$ & $\begin{array}{c}\operatorname{Mod}_{2} \\
(16)\end{array}$ \\
\hline aegis_001 & 1 & -1.000 & 0 & 0 & 23 & 1.992 & 1.907 & 2.068 & 1.818 & 2.148 & 95.066 & 30 & -99.00 & 0.00 & -999 \\
aegis_002_3 & 4 & -1.000 & 0 & 0 & 23 & 1.765 & 1.677 & 1.865 & 1.554 & 1.909 & 96.528 & 5 & -99.00 & 0.00 & -999 \\
aegis_003 & 5 & -1.000 & 0 & 0 & 23 & 1.485 & 1.396 & 1.954 & 1.334 & 1.996 & 54.259 & 7 & -99.00 & 0.00 & -999 \\
aegis_004_1 & 6 & -1.000 & 0 & 0 & 20 & 2.718 & 2.633 & 2.903 & 2.256 & 2.958 & 81.099 & 139 & -99.00 & 0.00 & -999 \\
aegis_005_3 & 10 & -1.000 & 0 & 0 & 23 & 1.472 & 1.436 & 1.766 & 1.412 & 1.822 & 75.805 & 139 & -99.00 & 0.00 & -999 \\
aegis_006_1 & 11 & -1.000 & 0 & 0 & 22 & 1.341 & 1.266 & 1.393 & 1.232 & 1.433 & 99.676 & 3 & -99.00 & 0.00 & -999 \\
aegis_007_2 & 14 & -1.000 & 0 & 0 & 15 & 2.567 & 2.098 & 3.147 & 1.472 & 3.383 & 50.924 & 4 & -99.00 & 0.00 & -999 \\
aegis_008 & 15 & 0.281 & 3 & 4 & 22 & 0.177 & 0.162 & 0.279 & 0.125 & 0.344 & 83.952 & 104 & -99.00 & 0.00 & -999 \\
aegis_009_1 & 16 & 0.769 & 2 & 2 & 22 & 0.747 & 0.713 & 0.784 & 0.692 & 0.873 & 92.742 & 121 & -99.00 & 0.00 & -999 \\
aegis_010 & 18 & -1.000 & 0 & 0 & 24 & 0.806 & 0.800 & 0.882 & 0.800 & 0.938 & 99.116 & 4 & -99.00 & 0.00 & -999
\end{tabular}

Note. Excerpt from the redshift catalog for AEGIS-X, which is published in its entirety in the HTML edition of the journal. Column 1: optical identifier number from the Rainbow catalog. Column 2: index number used to identify files in online database. Column 3: spectroscopic redshift, when available, otherwise -1 . Column 4: redshift confidence; we consider the spectroscopic redshift to be reliable when this value is 3 or greater. Column 5 : spectroscopic redshift reference, $0=$ no $\mathrm{z}_{\mathrm{spec}}$, $1=$ DEEP2+3 (Cooper et al. 2012; Newman et al. 2013), 2 = MMT (Coil et al. 2009; note, however, that this is the first time the redshifts themselves are published), $3=$ CFRS (Lilly et al. 1995), $4=$ SDSS (DR9, Ahn et al. 2012), $5=$ LBG (Steidel et al. 2003). Column 6: number of photometric points available for the fit. Column 7: photometric redshift defined as the primary peak of the $P(z)$ distribution. Columns 8 and 9: 68\% confidence lower and upper value of photometric redshift. Columns 10 and 11: $90 \%$ confidence lower and upper value of photometric redshift. Column 12: area under the curve $P(z)$ computed in the range $z_{p 1} \pm 0.1\left(1+z_{\mathrm{p} 1}\right)$, normalized to a percentage of the entire $P(z)$. Column 13: best fitting template; from 1 to 31 the templates are from S09, and templates from 100 + (1...30) are from the I09 library. Column 14: second solution for the photometric redshift, when $P\left(z_{\mathrm{p} 2}\right)$ exceeds $5 \%$, otherwise -99 . Column 15: same as column 12 , but for $z_{\mathrm{p} 2}$ if quoted in column 14, otherwise 0. Column 16: same as column 13, but for $z_{\mathrm{p} 2}$ if quoted in column 14, otherwise -999 .

(This table is available in its entirety in machine-readable form.)

coordinates of the sources and detection properties in the various X-ray bands. In the second table we provide X-ray properties as fluxes and hardness ratios. Each table is clearly described on the corresponding notes.

\section{A.2. Multiwavelength Catalog}

The multiwavelength properties of the counterparts are provided in Tables 13 and 14. In the first table we provide the coordinates of the counterparts in the optical and mid-infrared catalogs, together with their ID and offset from the X-ray coordinates. In the second table, for each counterpart we provide the magnitude in the $\mathrm{AB}$ system for all bands listed in Table 7. The photometry is corrected for Galactic extinction but not for zeropoint offset because this value is not independent of the SEDs used in its computation.

\section{A.3. Redshift Catalog}

The redshift catalog is shown in Table 15. For each of the sources we list the spectroscopic redshift if available, the redshift reliability and the source in the literature from which it originates. In addition, we provide the photometric redshift values and the $68 \%$ and $90 \%$ confidence limits, as well as a measure of the width of the peak of the photo-z probability distribution function $P(z)$, and the adopted template. If a significant second peak is seen in the $P(z)$ solution, details are also provided for this secondary solution. In general, we recommend adopting the full $P(z)$ rather than a single value for 
the photometric redshift. This is particularly important when the value of $P\left(z_{\mathrm{p} 1}\right)$ is low, which is often the case when the number of available photometric points is low or where a secondary solution exists. The full $P(z)$ and the templates used for fitting are available from the public web site http://www. mpe.mpg.de/XraySurveys.

\section{REFERENCES}

Aird, J. A., Coil, A. L., Moustakas, J., et al. 2012, ApJ, 746, 90 Aird, J. A., Nandra, K., Georgakakis, A., et al. 2008, MNRAS, 387, 883 Aird, J. A., Nandra, K., Laird, E. S., et al. 2010, MNRAS, 401, 2531 Ahn, C. P., Alexandroff, R., Allende Prieto, C., et al. 2012, ApJS, 203, 21 Alexander, D. M., Bauer, F. E., Brandt, W. N., et al. 2003, AJ, 126, 539 Barger, A. J., Cowie, L. L., Capak, P., et al. 2003, AJ, 126, 632 Barger, A. J., Cowie, L. L., Mushotzky, R. F., et al. 2005, AJ, 129, 578 Barro, G., Pérez-González, P. G., Gallego, J., et al. 2011a, ApJS, 193, 13 Barro, G., Pérez-González, P. G., Gallego, J., et al. 2011b, ApJS, 193, 30 Barmby, P., Alonso-Herrero, A., Donley, J. L., et al. 2006, ApJ, 642, 126 Barmby, P., Huang, J.-S., Ashby, M. L. N., et al. 2008, ApJS, 177, 431 Brandt, W. N., \& Hasinger, G. 2005, ARA\&A, 43, 827 Brightman, M., \& Nandra, K. 2012, MNRAS, 422, 1166 Brightman, M., Nandra, K., Salvato, M., et al. 2014, MNRAS, 443, 1999 Brightman, M., \& Ueda, Y. 2012, MNRAS, 423, 702

Brusa, M., Fiore, F., Santini, P., et al. 2009, å, 507, 1277

Brusa, M., Zamorani, G., Comastri, A., et al. 2007, ApJS, 172, 353 Buchner, J., Georgakakis, A., Nandra, K., et al. 2015, ApJ, 802, 89 Bundy, K., Georgakakis, A., Nandra, K., et al. 2008, ApJ, 681, 931 Cardamone, C. N., Urry, C. M., Damen, M., et al. 2008, ApJ, 680, 130 Cardamone, C. N., van Dokkum, P. G., Urry, C. M., et al. 2010, ApJS, 189,270

Ciliegi, P., Zamorani, G., Bondi, M., et al. 2005, A\&A, 441, 879

Ciliegi, P., Zamorani, G., Hasinger, G., et al. 2003, A\&A, 398, 901

Civano, F., Brusa, M., Comastri, A., et al. 2011, ApJ, 741, 91

Civano, F., Elvis, M., Brusa, M., et al. 2012, ApJS, 201, 30

Coil, A., Georgakakis, A., Newman, J. A., et al. 2009, ApJ, 701, 1484

Coil, A. L., Newman, J. A., Kaiser, N., et al. 2004, ApJ, 617, 765

Cooper, M. C., Aird, J. A., Coil, A. L., et al. 2011, ApJS, 193, 14

Cooper, M. C., Griffith, R. L., Newman, et al. 2012, MNRAS, 419, 3018

Dahlen, T., Mobasher, B., Faber, S. M., et al. 2013, ApJ, 775, 93

Davis, M., Faber, S. M., Newman, J., et al. 2003, SPIE, 4834, 161

Davis, M., Guhathakurta, P., Konidaris, N., et al. 2007, ApJL, 660, L1

Dickey, J. M., \& Lockman, F. J. 1990, ARA\&A, 28, 215

Elvis, M., Civano, F., Vignali, C., et al. 2009, ApJS, 184, 158

Erfanianfar, G., Finoguenov, A., Tanaka, M., et al. 2013, ApJ, 765, 117

Fiore, F., Puccetti, S., Grazian, A., et al. 2012, å, 537, 16

Fotopoulou, S., Salvato, M., Hasinger, G., et al. 2012, ApJS, 198, 1

Freeman, P. E., Kashyap, V., Rosner, R., \& Lamb, D. Q. 2002, ApJS, 138, 185

Fruscione, A., Burke, D., \& Siemiginowska, A. 2009, ChNew, 16, 26

Garmire, G. P., Bautz, M. W., Ford, P. G., Nousek, J. A., \& Ricker, G. R., Jr. 2003, Proc. SPIE, 4851, 28

Georgakakis, A., Coil, A. L., Laird, E. S., et al. 2009, MNRAS, 397, 623

Georgakakis, A., Gerke, B. F., Nandra, K., et al. 2008a, MNRAS, 391, 183

Georgakakis, A., Nandra, K., Laird, E. S., et al. 2007, ApJL, 660, L15
Georgakakis, A., Nandra, K., Laird, E. S., et al. 2008b, MNRAS, 388, 1205 Georgakakis, A., Nandra, K., Yan, R., et al. 2008c, MNRAS, 385, 2049 Georgakakis, A., et al. 2014a, MNRAS, 440, 339

Georgakakis, A., et al. 2014b, MNRAS, 443, 3327

Giacconi, R., Zirm, A., Wang, J., et al. 2002, ApJS, 139, 369

Grogin, N. A., Rajan, A., Donley, J. L., et al. 2011, ApJS, 197, 39

Goulding, A. D., Forman, W. R., Hickox, R. C., et al. 2012, ApJS, 202, 6

Hasinger, G., Cappelluti, N., Brunner, H., et al. 2007, ApJS, 172, 29

Hasinger, G., Miyaji, T., \& Schmidt, M. 2005, å, 441, 417

Hopkins, P. F., Hernquist, L., Martini, P., et al. 2005, ApJL, 625, L71

Hornschemeier, A. E., Bauer, F. E., Alexander, D. M., et al. 2003, AJ, 126, 575

Hornschemeier, A. E., Brandt, W. N., Garmire, G. P., et al. 2001, ApJ, 554,742

Hsu, L.-T., Salvato, M., Nandra, K., et al. 2014, ApJ, 796, 60

Ilbert, O., Arnouts, S., McCracken, H. J., et al. 2006, A\&A, 457, 841

Ilbert, O., Capak, P., Salvato, M., et al. 2009, ApJ, 690, 1236

Jerius, D., Cohen, L., Edgar, R., et al. 1995, BAAS, 27, 1395

Kenter, A., Murray, S. S., Forman, W. R., et al. 2005, ApJS, 161, 9

Kim, M., Kim, D., Wilkes, B. J., et al. 2007, ApJS, 169, 401

Kraft, R. P., Burrows, D. N., \& Nousek, J. A. 1991, ApJ, 374, 344

Koekemoer, A. M., Faber, S. M., Ferguson, H. C., et al. 2011, ApJS, 197, 36

Laird, E. S., Nandra, K., Georgakakis, A., et al. 2009, ApJS, 180, 102

Lehmer, B. D., Xue, Y. Q., Brandt, W. N., et al. 2012, ApJ, 752, 46

Lilly, S. J., le Fevre, O., Crampton, D., et al. 1995, ApJ, 455, 50

Luo, B., Brandt, W. N., Xue, Y. Q., et al. 2010, ApJS, 187, 560

Lusso, E., Comastri, A., Simmons, B., et al. 2012, MNRAS, 425, 623

Lusso, E., Comastri, A., Vignali, C., et al. 2010, å, 512, 34

Maccacaro, T., Caccianiga, A., et al. 1988, ApJ, 326, 680

Murray, S. S., Kenter, A., Forman, W. R., et al. 2005, ApJS, 161, 1

Nandra, K., Laird, E. S., Adelberger, K., et al. 2005, MNRAS, 356, 568

Nandra, K., O’Neill, P. M., George, I. M., et al. 2007, MNRAS, 382, 194

Newman, J. A., Cooper, M. C., Davis, et al. 2013, ApJS, 208, 5

Park, T., Kashyap, V. L., Siemiginowska, A., et al. 2006, ApJ, 652, 610

Pérez-González, P. G., Rieke, G. H., Villar, V., et al. 2008, ApJ, 675, 234

Pierce, C. M., Lotz, J. M., Laird, E. S., et al. 2007, ApJL, 660, L19

Pierre, M., Chiappetti, L., Pacaud, F., et al. 2007, MNRAS, 382, 279

Rangel, C., Nandra, K., Laird, E. S., et al. 2013, MNRAS, 428, 3089

Reach, W. T., Kuchner, M. J., von Hippel, T., et al. 2005, PASP, 117, 978

Salvato, M., Hasinger, G., Ilbert, O., et al. 2009, ApJ, 690, 1250

Salvato, M., Ilbert, O., Hasinger, G., et al. 2011, ApJ, 742, 61

Schmidt, M., Hasinger, G., Gunn, J., et al. 1998, A\&A, 329, 495

Silverman, J. D., Green, P. J., Barkhouse, et al. 2008, ApJ, 679, 118

Steidel, C. C., Adelberger, K. L., Shapley, A. E., et al. 2003, ApJ, 592, 728

Steidel, C. C., Shapley, A. E., Pettini, M., et al. 2004, ApJ, 604, 534

Szokoly, G. P., Bergeron, J., Hasinger, G., et al. 2004, ApJS, 155, 271

Tozzi, P., Gilli, R., Mainieri, V., et al. 2006, A\&A, 451, 457

Trouille, L., Barger, A. J., Cowie, L. L., et al. 2008, ApJS, 179, 1

Ueda, Y., Akiyama, M., Hasinger, G., et al. 2014, ApJ, 786, 104

Venemans, B. P., McMahon, R. G., Warren, S. J., et al. 2007, MNRAS, 376, 76

Vito, F., Vignali, C., Gilli, R., et al. 2013, MNRAS, 428, 354

Wall, J. V., Jackson, C. A., Shaver, P. A., et al. 2005, A\&A, 434, 133

Wang, J. X., Zheng, Z. Y., Malhotra, S., et al. 2007, ApJ, 669, 765

Whitaker, K. E., Labbé, I., van Dokkum, P. G., et al. 2011, ApJ, 735, 86

Xue, Y. Q., Luo, B., Brandt, W. N., et al. 2011, ApJS, 195, 10

Yang, Y., Mushotzky, R. F., Steffen, A. T., et al. 2004, AJ, 128, 1501 